THE ASTROPHYSICAL JOURNAL, 344:613-636, 1989 September 15

(C) 1989. The American Astronomical Society. All rights reserved. Printed in U.S.A.

\title{
MAJOR AND MINOR AXIS KINEMATICS OF 22 ELLIPTICALS ${ }^{1}$ Marijn FranX, ${ }^{2,3,4}$ GARTh Illingworth, ${ }^{3,4,5}$ AND Timothy HeCKMaN ${ }^{4,6}$ \\ Received 1988 December 5 ; accepted 1989 February 24
}

\begin{abstract}
Rotation curves and velocity dispersion profiles have been determined for the major and the minor axes of 22 elliptical galaxies. The uncertainty in the mean rotational velocity is typically only $5 \mathrm{~km} \mathrm{~s}^{-1}$ for either axis. Rotation $\left(\left\langle V_{r}\right\rangle \geq 20 \mathrm{~km} \mathrm{~s}^{-1}\right)$ was detected in all but one galaxy (NGC 5846), even though the sample was biased toward round ellipticals. Minor axis rotation larger than the major axis rotation was measured in two galaxies, NGC 4406 and NGC 7507. While the sample of such galaxies is still small (now three in total), it appears plausible that $\approx 10 \%$ of ellipticals may show large minor axis velocities relative to those on the major axis. Two more ellipticals, NGC 1549 and NGC 7145, show $v_{\text {minor }} \approx v_{\text {major }}$. In total six galaxies, or $27 \%$ of the sample, show significant minor axis rotation. A simple model is used to derive a rotational axis from the observed minor and major axis velocities to a typical accuracy of $6^{\circ}$. The rotational and photometric minor axes align to better than $10^{\circ}$ for $60 \%$ of the sample. This implies that the direction of the angular momentum is related to the orientation of the figure of the galaxy. This is surprising, since ellipticals are not supported by rotation, and considerable freedom in the direction of the angular momentum is allowed if galaxies are triaxial and have insignificant figure rotation. This suggests that figure rotation could play an important role in elliptical galaxies, or that these galaxies are near to oblate, and/or that events during the formation of elliptical galaxies conspire to orient the figure such that the angular momentum vector aligns approximately with the shortest of the principal axes. The galaxies with large photometric twists generally show significant kinematic misalignment. In addition, the centers of these galaxies are better aligned photometrically with their kinematic axes than are the outer parts. At least two galaxies have rapidly rotating, kinematically skew components in their cores. IC 1459 has a kinematically distinct core with its angular momentum opposite to the angular momentum of the outer parts, and NGC 4406 (a minor-axis rotator) has a core with its angular momentum perpendicular to that of the outer parts. The outer part of the latter galaxy may well rotate around the long axis. An additional four galaxies show weaker effects that need confirmation. The minor axis rotation and the misaligned cores support the view that ellipticals as a class are triaxial.
\end{abstract}

Subject headings: galaxies: evolution — galaxies: formation — galaxies: internal motions — galaxies: nuclei — galaxies: structure

\section{INTRODUCTION}

Our present-day understanding of bright ellipticals is that they have a triaxial shape and are supported by anisotropies in their velocity dispersion. The observational evidence for this is the slow rotation of bright ellipticals, too slow to account for their flattening (see e.g., Davies et al. 1983). Theoretical studies have shown that triaxial galaxies can exist in equilibrium (for a review, see de Zeeuw 1987), and that they form easily in $N$-body experiments (e.g., Wilkinson and James 1982).

Unfortunately, not very much more is known about their intrinsic shapes. The deprojection of the two-dimensional surface brightness distribution into the three-dimensional luminosity distribution is highly degenerate. This prohibits the determination of the intrinsic shapes of galaxies from surface photometry alone. For a few individual galaxies, the shapes are better constrained by the kinematics of the stars and gas. Studies of NGC 5128 (e.g., Wilkinson et al. 1986), NGC 1052 (Davies and Illingworth 1986), and NGC 5077 (Bertola et al.

\footnotetext{
${ }^{1}$ Partly based on observations made at the European Southern Observatory, La Silla, Chile.

${ }^{2}$ Sterrewacht Leiden, Leiden University.

${ }^{3}$ Space Telescope Science Institute, which is operated by AURA, Inc., for the National Aeronautics and Space Administration.

4 Visiting Astronomer at the Kitt Peak National Observatory and the Cerro Tololo Inter-American Observatory, operated by AURA, Inc., under contract to the National Science Foundation.

${ }^{5}$ Lick Observatory.

${ }^{6}$ Astronomy Program, University of Maryland.
}

1989) indicate that these galaxies are triaxial. The fraction of ellipticals with regular disks of ionized gas is too low, however, to use this method for the determination of the general distribution of the shapes of ellipticals.

Binney (1985) has shown how the stellar kinematics of ellipticals can be used to constrain their intrinsic shapes statistically. The rotation along the major and minor axis of ellipticals provides valuable information about their intrinsic shapes. In the first place, detection of minor axis rotation rules out the possibility (1) that ellipticals are oblate spheroids rotating about their short axis ("oblate rotators"). The assumption that they are (2) strongly prolate tumbling bars can also be tested, as can the view (3) that ellipticals rotate about their long axes. The limited data available to Binney were inconsistent with hypotheses (1), (2), and (3). Binney also showed how various hypotheses concerning the "triaxiality" of ellipticals could be tested against the observed ratios of minor axis-tomajor axis rotation. He noted that the available data suggested that ellipticals were "optimally triaxial," i.e., that they could be characterized as triaxial figures whose intermediate axis was truly intermediate between the longest and shortest axes. This result, however, is very uncertain, either because the rotation curves are poorly determined or because the galaxies themselves are unusual in their photometric and kinematical properties. Examples of such galaxies with multiaxis velocity profiles would be NGC 596 (Schechter and Gunn 1979; Williams 1981) and NGC 4125 (Bertola et al. 1984).

We have started a program to measure the major and minor 
axis rotation curves of a large sample of ellipticals. This paper is the second in a series of papers. Paper I (Franx, Illingworth, and Heckman 1989) described two-dimensional multicolor surface photometry on a sample of galaxies. Here we present kinematical observations on 22 elliptical galaxies in the northern and southern hemisphere. The results will be analyzed in a third paper (Franx, Illingworth and de Zeeuw 1989, hereafter FIZ). A preliminary discussion of the results is given by Franx (1988).

The paper is organized as follows. The sample is discussed in $\S$ II. The observations are described in $\S$ III. Section IV deals with the data reduction, and the derivation of the rotational velocities and velocity dispersions. The resulting rotation curves and velocity dispersion profiles are given in $\S \mathrm{V}$. The implications of these results are discussed in $\S \mathrm{VI}$.

Those readers interested in the results would be advised to skip initially to $\S \mathrm{V}$ from $\S$ II or $\S$ III. Section IV is lengthy, because of the variety of detectors and spectrographs used and the difficulties encountered in reducing some of the data.

\section{SAMPLE SELECTION}

A sample of galaxies with accurately known position angles and ellipticities was required for the spectroscopic observations. We compiled a list of galaxies from Paper I and the photometric studies of Davis et al. (1985), Djorgovski (1985), Lauer (1985), Jedrzejewski (1987), and Peletier et al (1989). Using these data and galaxy classifications taken from the RC2 (de Vaucouleurs, de Vaucouleurs, and Corwin 1976), a set of elliptical galaxies was selected based on apparent size, absolute magnitude, and ellipticity. Large, round galaxies with an absolute magnitude $M_{B}$ between -20 and -22 were given the highest priority. We assume here, and in the following, a value of $H_{0}$ of $50 \mathrm{~km} \mathrm{~s}^{-1} \mathrm{Mpc}^{-1}$. Round galaxies were given high priority because Binney's (1985) models predict that apparently round galaxies have the highest chance of showing minor axis rotation that is a measurable fraction of the major axis rotation. Furthermore, there is a scarcity of kinematical observations of round galaxies.

The observed galaxies are listed in Table 1, with classifications, total magnitudes, length scales, mean ellipticities, position angles, group velocities, central velocity dispersions, visible luminosities, and $I R A S 100 \mu \mathrm{m}, \mathrm{X}$-ray, and radio fluxes. The sample is by no means complete in any parameter, partly because there is no complete all-sky sample of ellipticals with accurate two-dimensional surface photometry, and partly because of the bias toward round galaxies noted above. The galaxies were selected irrespective of their radio fluxes, X-ray properties and of their $100 \mu \mathrm{m} I R A S$ fluxes, and thus should have radio, X-ray, and far-IR properties characteristic of nearby elliptical galaxies, except for those ellipticals that were known to be very dusty or quite unusual. These were excluded from our sample because of the difficulty of deriving photometric and kinematic properties. Some of the galaxies were subsequently found to have (weak) shells or dust features.

\section{OBSERVATIONS}

Our long-slit spectroscopic observations were taken with the $4 \mathrm{~m} \mathrm{KPNO}$ and CTIO telescopes, and the $2.2 \mathrm{~m}$ and $3.6 \mathrm{~m}$ ESO telescopes on nine usable nights between 1984 and 1987. A wide variety of detectors were used. At KPNO, we used the RC Spectrograph with the ICCD (Intensified CCD) detector. At CTIO the RC Spectrograph was used in combination with

TABLE 1

OBSERVED Galaxies

\begin{tabular}{|c|c|c|c|c|c|c|c|c|c|c|c|c|c|c|c|}
\hline \multirow{2}{*}{$\begin{array}{c}\text { Galaxy } \\
\text { (1) }\end{array}$} & \multicolumn{2}{|c|}{ Classification } & \multirow{2}{*}{$\begin{array}{l}B_{T} \\
\\
(4)\end{array}$} & \multirow{2}{*}{$\begin{array}{l}r_{e} \\
" \prime \\
(5)\end{array}$} & \multirow{2}{*}{$\begin{array}{l}r_{25} \\
\prime \\
(6)\end{array}$} & \multirow[b]{2}{*}{$\begin{array}{c}r_{n} \\
\prime \prime \\
(7)\end{array}$} & \multirow{2}{*}{$\begin{array}{c}\epsilon \\
(8)\end{array}$} & \multirow{2}{*}{$\begin{array}{r}\widehat{\Theta}_{m a j} \\
\quad(9)\end{array}$} & \multirow{2}{*}{$\begin{array}{c}v_{g r p} \\
\mathrm{~km} \mathrm{~s}^{-1} \\
(10)\end{array}$} & \multirow{2}{*}{$\begin{array}{c}\sigma \\
\mathrm{km} \mathrm{s}^{-1} \\
(11)\end{array}$} & \multirow{2}{*}{$\begin{array}{l}M_{B} \\
(12)\end{array}$} & \multirow{2}{*}{$\begin{array}{c}\text { scale } \\
\mathrm{kpc}^{-1} \\
\operatorname{arcsec}^{-1} \\
\quad(13)\end{array}$} & \multirow{2}{*}{$\begin{array}{r}\mathrm{F}_{100 \mu m} \\
\mathrm{mJy}\end{array}$} & \multirow{2}{*}{$\begin{array}{r}\mathrm{F}_{X} \\
10^{13} \mathrm{ergs} \\
\mathrm{cm}^{-2} \mathrm{~s}^{-1} \\
(15)\end{array}$} & \multirow{2}{*}{$\begin{array}{r}\mathrm{F}_{\text {Radio }} \\
\mathrm{mJy}\end{array}$} \\
\hline & $\mathrm{RC} 2$ & RSA & & & & & & & & & & & & & \\
\hline NGC 636 & $\mathrm{E} 3 / \mathrm{E} 1$ & E0 & 12.33 & 19 & 60 & 40 & 0.15 & 52 & 1865 & 156 & -20.53 & 0.18 & & & $<5$ \\
\hline NGC 1379 & E0 & E0 & 11.66 & 42 & 93 & 21 & 0.03 & 4 & 1411 & 133 & -20.59 & 0.14 & & & $<6$ \\
\hline NGC 1395 & E2/E3 & $\mathrm{E} 2$ & 10.94 & 45 & 122 & 34 & 0.18 & 94 & 1585 & 258 & -21.57 & 0.15 & 390 & 6.92 & $<15$ \\
\hline NGC 1399 & E1P & $\mathrm{E} 1$ & 10.55 & 42 & 137 & 45 & 0.11 & 110 & 1411 & 310 & -21.70 & 0.14 & 340 & $\ldots$ & 342 \\
\hline NGC 1404 & E1 & E2 & 10.89 & 43 & 106 & 27 & 0.12 & 173 & 1411 & 225 & -21.36 & 0.14 & 290 & $\ldots$ & $<18$ \\
\hline NGC 1407 & $\mathrm{E} 0 / \mathrm{E} 0$ & E0 & 10.57 & 72 & 154 & 34 & 0.05 & 56 & 1585 & 285 & -21.94 & 0.15 & 480 & 11.72 & 44 \\
\hline NGC 1439 & $\mathrm{E} 1 / \mathrm{E} 1$ & E1 & 12.07 & 41 & 79 & 16 & 0.09 & 33 & 1585 & 156 & -20.44 & 0.15 & & $\ldots$ & $<18$ \\
\hline NGC 1549 & E0 & $\mathrm{E} 2$ & 10.58 & 47 & 140 & 42 & 0.13 & 120 & 1162 & 205 & -21.25 & 0.11 & 180 & $\ldots$ & $<13$ \\
\hline NGC 1700 & $\mathrm{E} 4 / \mathrm{E} 1 \mathrm{~T}$ & E3 & 12.26 & 14 & 56 & 24 & 0.28 & 89 & 4082 & 233 & -22.30 & 0.40 & $<654$ & $\ldots$ & $<3$ \\
\hline NGC 3091 & E3/E2 & E3 & 12.09 & 31 & 74 & 19 & 0.27 & 147 & 3612 & 290 & -22.20 & 0.35 & & & $<10$ \\
\hline NGC 3379 & E1/E1 & E0 & 10.43 & 35 & 134 & 52 & 0.10 & 70 & 792 & 201 & -20.57 & 0.08 & $<327$ & $<3.2$ & 1.05 \\
\hline NGC 3557 & $\mathrm{E} 3$ & E3 & 11.13 & 38 & 109 & 33 & 0.25 & 33 & $3014^{\mathrm{a}}$ & 292 & -22.77 & 0.29 & 750 & & 290 \\
\hline NGC 4406 & E3/E3 & E3 & 9.87 & 90 & 208 & 52 & 0.21 & 121 & 1153 & 250 & -21.94 & 0.11 & $\ldots$ & 47.50 & $<12$ \\
\hline NGC 4472 & $\mathrm{E} 2 / \mathrm{E} 4$ & $\mathrm{E} 1 / \mathrm{S} 0$ & 9.32 & 104 & 261 & 69 & 0.17 & 162 & 1153 & 250 & -22.49 & 0.11 & .. & 65.31 & $<132$ \\
\hline NGC 4649 & $\mathrm{E} 2 / \mathrm{E} 1$ & So & 9.77 & 74 & 208 & 60 & 0.15 & 103 & 1153 & 341 & -22.04 & 0.11 & & 31.67 & 19.5 \\
\hline IC 4296 & E0 & E0 & 11.29 & 57 & 111 & 24 & 0.10 & 59 & 3713 & 323 & -23.06 & 0.36 & $<450$ & 4.02 & 6410 \\
\hline NGC 5846 & $\mathrm{E} 0 / \mathrm{E} 0$ & S0 & 10.67 & 83 & 150 & 29 & 0.08 & 71 & 1674 & 278 & -21.95 & 0.16 & & 36.04 & 20 \\
\hline NGC 7144 & E0 & E0 & 11.75 & 40 & 89 & 21 & 0.05 & 20 & 1861 & 185 & -21.10 & 0.18 & 330 & $\ldots$ & $<7$ \\
\hline NGC 7145 & E0 & E0 & 11.98 & 39 & 81 & 18 & 0.05 & 125 & $\cdot 1861$ & 132 & -20.87 & 0.18 & & & $<4$ \\
\hline IC 1459 & E3 & E4 & 10.88 & 39 & 119 & 38 & 0.27 & 38 & 1647 & 308 & -21.71 & 0.16 & 450 & 3.31 & 887 \\
\hline NGC 7507 & $\mathrm{E} 0 / \mathrm{E} 0$ & E0 & 11.15 & 31 & 104 & 35 & 0.04 & 100 & 1553 & 238 & -21.31 & 0.15 & $<459$ & & $<10$ \\
\hline NGC 7619 & $\mathrm{E} 2 / \mathrm{E} 1$ & E3 & 12.00 & 32 & 77 & 20 & 0.22 & 35 & 3493 & 337 & -22.22 & 0.34 & $\ldots$ & 7.12 & 22 \\
\hline
\end{tabular}

Notes.-Col. (2) and col. (3) give the classifications of the galaxies from de Vaucouleurs et al. 1976 (RC2) and Sandage and Tammann 1981 (RSA). Cols. (4), (5), (6), and (7) give the total $B$ magnitude $B_{T}$, the effective radius $r_{e}$, the radius $r_{25}$ at which $\mu_{B}=25 \mathrm{mag}$ arcsec ${ }^{-2}$, and the radius $r_{n}$, defined by Burstein et al 1987 . The values listed in these columns are taken from Burstein et al. 1987 . The ellipticity and position angle in cols. (8) and (9) are the average between $10^{\prime \prime}$ and $30^{\prime \prime}$ and are derived from the surface photometry of Franx, Illingworth, and Heckman 1989, Lauer 1985, Jedrzejewski 1987, and Peletier et al. (1989). The group velocity and galaxy dispersion in cols. (10) and (11) are taken from Davies $e$ t al. 1987. The absolute magnitude $M_{B}$ is calculated with a value for $H_{0}$ of $50 \mathrm{~km} \mathrm{~s}^{-1} \mathrm{Mpc}^{-1}$. The IRAS $100 \mu \mathrm{m}$ fluxes in col. (14) are taken from Jura et al. 1987, the X-ray fluxes in col. (15) are taken from Canizares et al. (1987), and the radio fluxes in col. (16) are taken from Disney and Wall 1977, Dressel and Condon 1978, and Birkinshaw and Davies (1985)

a Velocity of galaxy. 
TABLE 2

InStrumental Parameters

\begin{tabular}{|c|c|c|c|c|}
\hline \multirow[b]{2}{*}{ PARAMETER } & \multicolumn{4}{|c|}{ OBSERVATORY } \\
\hline & KPNO & CTIO & ESO & ESO \\
\hline ……… & 1984 Nov, 1985 Dec & 1985 Oct, 1986 Nov & 1986 Oct & $1987 \mathrm{Apr}$ \\
\hline Telescope (m) .............. & 4 & 4 & 2.2 & 3.6 \\
\hline Spectrograph ........ & RC & RC & $\mathrm{B} \& \mathrm{C}$ & $\mathrm{B} \& \mathrm{C}$ \\
\hline Wavelengths $(\dddot{\AA}) \ldots . .$. & $3700-4700$ & $4100-5700$ & $4750-5610$ & $4740-5620$ \\
\hline Detector ....................... & ICCD & 2D Frutti & RCA CCD & RCA CCD \\
\hline Format $\ldots \ldots \ldots \ldots \ldots \ldots$ & $800 \times 800$ & $512 \times 1520$ & $320 \times 512$ & $640 \times 1024$ \\
\hline Noise & $16 e^{-}$ & $\ldots$ & $80 e^{-}$ & $40 e^{-}$ \\
\hline Dispersion $\left(\AA\right.$ pixel $\left.^{-1}\right) \ldots \ldots$ & 1.3 & 1.1 & 1.7 & 0.9 \\
\hline Scale $\left(\operatorname{arcsec}\right.$ pixel $\left.^{-1}\right) \ldots \ldots$ & 0.86 & 0.68 & 1.8 & 0.5 \\
\hline Resolution $(\sigma)\left(\mathrm{km} \mathrm{s}^{-1}\right) \ldots$ & 110 & 90 & 200 & 50 \\
\hline
\end{tabular}

the 2D-Frutti, a photon counting system. At ESO we used the Boller and Chivens Spectrographs with RCA CCDs at both telescopes. Details of the instrumental setups are given in Table 2.

The scale along the slit varied from 0.5 pixel $^{-1}$ at the ESO $3.6 \mathrm{~m}$ to 1.8 pixel $^{-1}$ at the ESO $2.2 \mathrm{~m}$ telescope. The spatial resolution of our data ranged from $1^{\prime \prime}$ at best to $2^{\prime \prime}-3$ " at the 2.2 $\mathrm{m}$. At KPNO, our data covered the wavelength interval from 3700 to $4700 \AA$, and included the $H$ and $K$ band, the $G$ band, and a variety of features to the red of the $G$ band. At CTIO, the spectral range was 4100 to $5700 \AA$, including the $G$ band and the $5170 \AA$ magnesium $b$-line triplet. The ESO observations were centered on the magnesium triplet, and covered the range from 4750 to $5610 \AA$. The dispersion was typically $1 \AA$ pixel $^{-1}$. The wavelength resolution varied from 2 to $3 \AA$ FWHM, equivalent to velocity dispersions of $50-110 \mathrm{~km} \mathrm{~s}^{-1}$, except for the $2.2 \mathrm{~m}$ telescope data, which had a resolution equivalent to $\sigma \approx 200 \mathrm{~km} \mathrm{~s}^{-1}$.

The observations are listed in Table 3, where the observing site, the position angles, the axis, major or minor, and the integration time are noted. The procedures followed depended upon the detector. At KPNO, we made a series of 300-500 s exposures to avoid saturation of the CCD. These typically totalled 2000-3000 s. The low readout noise of the CCD, and the high photon gain of the image intensifier ensured that we were not at all limited by readout noise or any charge transfer problems. At the beginning and end of each series a $\mathrm{HeNeAr}$ arc lamp exposure was taken for geometric calibration purposes. At CTIO, we took one to two 1500-2000+ s exposures that totalled $3000-5000+s$ per galaxy axis. The galaxy centers were shifted after each exposure, to minimize the influence of detector "glitches" in the processed data. HeNeAr calibration exposures were again taken before and after each series. The KPNO ICCD and the 2D-Frutti suffered from saturation effects at the high surface brightnesses in the centers of these bright galaxies. We took some short exposures (ICCD) or exposures using a smaller slit width (2D-Frutti) to minimize these effects.

At ESO, we were limited by the relatively high readout noise of the RCA CCDs, and so it was important to maximize the integration time per exposure. It proved to be optimum to take separate exposures for the cores and the outer parts. Integration times of up to $2400 \mathrm{~s}$ were used for the outer parts. At the $3.6 \mathrm{~m}$ the pixels were binned $4 \times 1$ on-chip along the spatial direction to increase the $\mathrm{S} / \mathrm{N}$ ratio. A wide $3^{\prime \prime}$ slit was used for these exposures. Short exposures, with the full spatial resolution, were taken on the cores with a narrow slit, compa- rable to the seeing $\left(1^{\prime \prime}-1\right.$ ".5). Calibration exposures were taken less often, because the instrument proved to be very stable. Template stars were observed each night. Flat-field frames and dark frames were taken during the day, particularly for the 2D-Frutti for which flat-field integrations in excess of 15,000 20,000 s were required.

\section{REDUCTION}

The data reduction consisted of two steps: first, photometric and geometric calibrations were performed to transform the data into a geometrically rectified form from which the sky was subtracted, and second, radial velocities and velocity dispersions were determined from analysis of the galaxy spectrum and a standard star spectrum. These steps are discussed separately. Those readers interested in the results should leave this section to later and move on to $\S \mathrm{V}$.

\section{a) Calibration of the Spectra}

The calibration process was carried out on the Leiden Observatory VAX computer, with the help of the IRAF package, kindly made available by NOAO. The general reduction process consists, in the order presented, of the following steps: (1) subtraction of bias, dark current and/or scattered light; (2) flat-field division; (3) correction for bad pixels in the detector and/or removal of cosmic-ray events; (4) wavelength calibration and geometrical rectification of the image; (5) determination and correction of the two-dimensional largescale response of the spectrograph and the detector; and (6) sky subtraction.

The end product was a two-dimensional spectrum, binned logarithmically in the wavelength direction and linearly in the spatial direction. In the following, because of the quite distinct characteristics of the different detector systems, we discuss aspects of the reduction of the KPNO, CTIO and ESO data separately.

$$
\text { i) ICCD Data }
$$

The ICCD consisted of an RCA magnetically focused C33063 image intensifier, optically coupled to a T1 $800 \times 800$ CCD array. The system was operated in a high-gain integrating mode, and not as a photon-event counting system. It thus suffered from a significant level of scattered light, that could be identified on the regions of the CCD which were not exposed directly by the spectrograph. The scattered light was caused by scattering in the image intensifier. To correct for this effect, a spline curve was fitted to an average of the columns at one side of the CCD which were exposed only to the scattered 
TABLE 3

OBSERVATION LoG

\begin{tabular}{|c|c|c|c|c|}
\hline $\begin{array}{l}\text { Galaxy } \\
\text { (1) }\end{array}$ & $\begin{array}{c}\operatorname{Run}^{\mathrm{a}} \\
(2)\end{array}$ & $\begin{array}{l}\mathbf{P A}^{\mathrm{b}} \\
(3)\end{array}$ & $\begin{array}{c}\text { Axis }^{\mathrm{c}} \\
(4)\end{array}$ & $\begin{array}{l}\text { Exposures }^{d} \\
\quad(5)\end{array}$ \\
\hline NGC 636 & K84 & $\begin{array}{r}60 \\
150\end{array}$ & $\begin{array}{l}\text { Major } \\
\text { Minor }\end{array}$ & $\begin{array}{l}5 \times 500 \mathrm{~s} \\
600 \mathrm{~s}, 7 \times 500 \mathrm{~s}\end{array}$ \\
\hline NGC 1379 ......... & $\mathrm{C} 86$ & $\begin{array}{r}7 \\
97\end{array}$ & $\begin{array}{l}\text { Major } \\
\text { Minor }\end{array}$ & $\begin{array}{l}2000 \mathrm{~s}, 2400 \mathrm{~s} \\
2 \times 2000 \mathrm{~s}\end{array}$ \\
\hline NGC 1395 & K84 & $\begin{array}{r}93 \\
3\end{array}$ & $\begin{array}{l}\text { Major } \\
\text { Minor }\end{array}$ & $\begin{array}{l}300 \mathrm{~s}, 4 \times 500 \mathrm{~s} \\
8 \times 500 \mathrm{~s}\end{array}$ \\
\hline NGC 1399 & $\mathrm{C} 86$ & $\begin{array}{r}115 \\
25\end{array}$ & $\begin{array}{l}\text { Major } \\
\text { Minor }\end{array}$ & $\begin{array}{l}3000 \mathrm{~s}, 3000 \mathrm{~s} \\
4000 \mathrm{~s}\end{array}$ \\
\hline NGC $1404 \ldots \ldots \ldots$ & $\mathrm{C} 86$ & $\begin{array}{r}164 \\
74\end{array}$ & $\begin{array}{l}\text { Major } \\
\text { Minor }\end{array}$ & $\begin{array}{l}2 \times 2000 \mathrm{~s} \\
2 \times 2000 \mathrm{~s}, 600 \mathrm{~s}\end{array}$ \\
\hline NGC $1407 \ldots$ & $\mathrm{C} 86$ & $\begin{array}{r}58 \\
148\end{array}$ & $\begin{array}{l}\text { Major } \\
\text { Minor }\end{array}$ & $\begin{array}{l}2 \times 1500 s \\
2 \times 2000 s\end{array}$ \\
\hline NGC $1439 \ldots \ldots \ldots$ & $\mathrm{C} 86$ & $\begin{array}{r}38 \\
128\end{array}$ & $\begin{array}{l}\text { Major } \\
\text { Minor }\end{array}$ & $\begin{array}{l}2 \times 1800 \mathrm{~s} \\
2000 \mathrm{~s}, 2500 \mathrm{~s}, 900 \mathrm{~s}\end{array}$ \\
\hline NGC $1549 \ldots$ & $\mathrm{C} 86$ & $\begin{array}{r}127 \\
37\end{array}$ & $\begin{array}{l}\text { Major } \\
\text { Minor }\end{array}$ & $\begin{array}{l}2 \times 1500 \mathrm{~s} \\
2 \times 2000 \mathrm{~s}\end{array}$ \\
\hline NGC 1700 & K84 & $\begin{array}{r}91 \\
1\end{array}$ & $\begin{array}{l}\text { Major } \\
\text { Minor }\end{array}$ & $\begin{array}{l}3 \times 500 \mathrm{~s}, 5 \times 300 \mathrm{~s} \\
5 \times 300 \mathrm{~s}, 3 \times 300 \mathrm{~s}\end{array}$ \\
\hline NGC 3091 & E87 & $\begin{array}{r}147 \\
57\end{array}$ & $\begin{array}{l}\text { Major } \\
\text { Minor }\end{array}$ & $\begin{array}{l}1200 \mathrm{~s}, 2400 \mathrm{~s}, 1800 \mathrm{~s} \\
1200 \mathrm{~s}, 2400 \mathrm{~s}\end{array}$ \\
\hline NGC 3379 & K84 & $\begin{array}{r}68 \\
158\end{array}$ & $\begin{array}{l}\text { Major } \\
\text { Minor }\end{array}$ & $\begin{array}{l}7 \times 300 \mathrm{~s}, 5 \times 150 \mathrm{~s} \\
9 \times 300 \mathrm{~s}, 2 \times 150 \mathrm{~s}\end{array}$ \\
\hline NGC $3557 \ldots \ldots \ldots$ & E87 & $\begin{array}{r}33 \\
35 \\
129\end{array}$ & $\begin{array}{l}\text { Major } \\
\text { Major } \\
\text { Minor }\end{array}$ & $\begin{array}{l}2700 \mathrm{~s} \\
1800 \mathrm{~s} \\
900 \mathrm{~s}, 2700 \mathrm{~s}\end{array}$ \\
\hline NGC 4406 & E87 & $\begin{array}{r}120 \\
30\end{array}$ & $\begin{array}{l}\text { Major } \\
\text { Minor }\end{array}$ & $\begin{array}{l}2400 \mathrm{~s}, 1200 \mathrm{~s} \\
2400 \mathrm{~s}\end{array}$ \\
\hline NGC 4472 . & E87 & $\begin{array}{r}162 \\
72\end{array}$ & $\begin{array}{l}\text { Major } \\
\text { Minor }\end{array}$ & $\begin{array}{l}3 \times 1200 \mathrm{~s} \\
1800 \mathrm{~s}\end{array}$ \\
\hline NGC $4649 \ldots \ldots \ldots$ & E87 & $\begin{array}{r}102 \\
12\end{array}$ & $\begin{array}{l}\text { Major } \\
\text { Minor }\end{array}$ & $\begin{array}{l}2700 s \\
2700 s\end{array}$ \\
\hline IC $4296 \ldots$ & E87 & $\begin{array}{r}65 \\
60\end{array}$ & $\begin{array}{l}\text { Major } \\
\text { Major }\end{array}$ & $\begin{array}{l}1200 \mathrm{~s} \\
2100 \mathrm{~s}\end{array}$ \\
\hline NGC $5846 \ldots$ & E87 & $\begin{array}{r}150 \\
80 \\
170\end{array}$ & $\begin{array}{l}\text { Minor } \\
\text { Major } \\
\text { Minor }\end{array}$ & $\begin{array}{l}2 \times 1800 \mathrm{~s}, 2300 \mathrm{~s}, 550 \mathrm{~s}, 1550 \mathrm{~s} \\
2700 \mathrm{~s}, 3600 \mathrm{~s} \\
440 \mathrm{~s}, 3600 \mathrm{~s}\end{array}$ \\
\hline NGC $7144 \ldots$. & $\mathrm{C} 86$ & $\begin{array}{r}27 \\
117\end{array}$ & $\begin{array}{l}\text { Major } \\
\text { Minor }\end{array}$ & $\begin{array}{l}2 \times 1500 \mathrm{~s} \\
2 \times 2000 \mathrm{~s}\end{array}$ \\
\hline NGC $7145 \ldots$ & $\mathrm{C} 86$ & $\begin{array}{r}132 \\
37\end{array}$ & $\begin{array}{l}\text { Major } \\
\text { Minor }\end{array}$ & $\begin{array}{l}1500 \mathrm{~s}, 2500 \mathrm{~s} \\
2 \times 2000 \mathrm{~s}\end{array}$ \\
\hline IC $1459 \ldots \ldots$ & $\begin{array}{l}\text { E86 } \\
\text { C86 }\end{array}$ & $\begin{array}{r}39 \\
39 \\
129\end{array}$ & $\begin{array}{l}\text { Major } \\
\text { Major } \\
\text { Minor }\end{array}$ & $\begin{array}{l}2700 \mathrm{~s} \\
2 \times 2000 \mathrm{~s}, 900 \mathrm{~s}, 700 \mathrm{~s}, 1200 \mathrm{~s}, 900 \mathrm{~s} \\
2 \times 2000 \mathrm{~s}, 1000 \mathrm{~s}, 2 \times 900 \mathrm{~s}\end{array}$ \\
\hline & E87 & 39 & Major & $1200 \mathrm{~s}$ \\
\hline NGC $7507 \ldots \ldots \ldots$ & $\mathrm{C} 86$ & $\begin{array}{r}105 \\
15\end{array}$ & $\begin{array}{l}\text { Major } \\
\text { Minor }\end{array}$ & $\begin{array}{l}1200 \mathrm{~s}, 2 \times 1500 \mathrm{~s} \\
2000 \mathrm{~s}, 900 \mathrm{~s}, 1200 \mathrm{~s}, 1200 \mathrm{~s}\end{array}$ \\
\hline NGC 7619 ......... & K85 & $\begin{array}{r}34 \\
124\end{array}$ & $\begin{array}{l}\text { Major } \\
\text { Minor }\end{array}$ & $\begin{array}{l}900 \mathrm{~s}, 4 \times 500 \mathrm{~s} \\
8 \times 500 \mathrm{~s}\end{array}$ \\
\hline
\end{tabular}

a Observatory and year of observation (C = CTIO, E = ESO, $\mathrm{K}=\mathrm{KPNO})$.

b Position angle.

c Nominal axis.

d Exposure sequences.

light. The fit was subtracted from the whole spectrum. Smooth surfaces were fitted to the flat fields, and the flat fields were normalized by the fits, to obtain a map of the response variations on small scales. The small-scale response variations proved to be constant throughout the run, and so a mean response frame was divided into all of the exposures. Some columns with bad charge transfer or hot pixels were identified, and removed by interpolation. Cosmic-ray events were not removed in these short exposures because of their rarity.

Following this photometric rectification, relative shifts and tilts of the data frames were determined as part of the geometric rectification. To that end, the $4358 \AA \mathrm{Hg}$ nightsky line was mapped in the object frames, and the $4471 \AA \mathrm{He}$ I line was mapped in the HeNeAr calibration frames. Shifts between exposures at the same hour angle, declination and position angle were small ( $\approx 0.5$ pixel or less), but a change of position angle could induce easily a shift of 1 pixel in the wavelength direction, or $15 \mu \mathrm{m}$. Using the night-sky line and the calibration arc line positions, we established a series of consecutive $\mathrm{HeNeAr}$ exposures for which the shifts were small (less than 1 pixel); these were averaged to give high $\mathrm{S} / \mathrm{N}$ line spectra for mapping the distortions. We mapped 21 emission lines along the slit in the averaged frames. The geometrical distortion due to the spectrograph and image intensifier tube was relatively small, the lines being curved by less than 1 pixel. A twodimensional polynomial was used to fit the dependence of 
wavelength as a function of position on the detector. The residuals from the fit were again small, being about $0.2 \AA$, equivalent to $15 \mathrm{~km} \mathrm{~s}^{-1}$ per fitted point on a spectral line. Absorption-line velocities could be derived with higher accuracy because the smooth fit averaged out the errors caused by noise, and because the line features were distributed throughout the spectrum. Similarly, the centers of the galaxies were traced in the wavelength direction to map out the $S$-distortion, and a polynomial was fitted. Finally, the two solutions were inverted, and the images were rectified and rebinned onto a logarithmic wavelength scale in one step.

The next step was to determine and remove the large-scale (low spatial frequency) variations in the throughput of the detector/spectrograph combination. Night sky exposures made during the cloudy parts of the nights were used for this. Each sky exposure was averaged along the slit to a onedimensional spectrum, which was then divided into the original two-dimensional spectrum to remove most of the spectral structure in the frame. These ratio frames were similar throughout the run, except for a gradual increase of the background level in the center of the slit. This increase was apparently caused by the now well-known "residual image" hysteresis effect of the TI CCD. The saturated bright galactic nuclei left residual charge that contaminated the sky frames. The residual charge accumulation during a sky frame was typically at the $10 \%$ level along the rows illuminated by the galaxy nuclei. Since the effect was significant only in a few rows of the CCD image near the center of the slit, we countered it by interpolating across the affected region. Smooth surfaces were then fitted to these processed sky frames to give the frames that characterized the low spatial frequency response of the system; the galaxy frame was then divided by the appropriate response frame. Note that we did not calibrate the absolute response as a function of wavelength, and so the images still contained the smooth wavelength-dependent spectrograph/detector response variations.

Finally, the night sky spectrum was determined for each galaxy image from the outer ends of the spectrum, where night sky emission and scattered solar light dominated. This sky spectrum was then subtracted from the image. The accuracy of the sky subtraction was determined by the accuracy of the calibration of the large-scale response of the system. The main problem here was the contribution of scattered light. Clearly the response function will be in error if we systematically overor underestimate the scattered light; we estimate that the accuracy with which we could determine the large-scale response was $5 \%$.

\section{ii) 2 D-Frutti Data}

The reduction of the 2D-Frutti data followed the same path, but was complicated by detector effects. The $2 \mathrm{D}$-Frutti is a photon event-counting system consisting of the same RCA C33063 image intensifier as is used in the ICCD, lens-coupled to a Varo Electrostatic intensifier tube and a microchannel plate combination. The output of the channel plate is fibercoupled to a Fairchild CCD, which is read out at a clock rate of $15 \mathrm{MHz}$. A $3 \times 3$ pixel photon discriminator algorithm locates photons to $\frac{1}{4}$ of a CCD pixel along both the wavelength and spatial direction; thus the $128 \times 380$ format of the CCD maps into a final format of $512 \times 1520$.

We found a relatively high level of scattered light in the inner part of the field of the detector. This was quite apparent outside the region illuminated by the slit. In addition, the response of the detector was found to decrease significantly at both the high- and low-wavelength ends of the spectrum, dropping to $20 \%$ of the response at the center. This decrease was also found in the dark exposures, and was thus due to the detector, and not to the spectrograph. We believe that both effects have the same cause, and result from the photon gain varying quite significantly over the field of the detector chain. With a fixed discriminator level for the photon events one is forced to a compromise which loses real photon events at the edges of the field where the gain is lowest, while accepting more dark events and scattered light events than desired in the central regions. For such a detector chain it is highly desirable to have discriminator settings that vary dynamically.

Another problem was the existence of a band covering $\approx 10 \%$ of the width of the frame and running along the length of the spectrum that was of lower intensity in the middle of the image. This band was subsequently found to be a region in which the background level was lower. It arose as a result of additional dark and scattered light events in the regions to either side of the band. These additional events were due to the on-chip buffering approach that was used as part of the readout process. The whole CCD was not read out following each frame cycle, but only the subset of the rows that corresponded to the portion of the CCD illuminated by the slit (128 rows for our slit size). The format of the CCD was such that only the central 128 of the 240 rows were illuminated directly by the slit, leaving 56 rows on either side. At the end of each $10 \mathrm{~ms}$ frame period, 128 rows were clocked out. Thus, of the rows containing data, only the $128-56=72$ rows nearest the serial register were actually clocked out. The remaining 56 rows containing data from the previous frame period lay adjacent to the then currently illuminated area, where they were susceptible to scattered light and where further dark events could accumulate. For galaxy frames, the contribution was comparable for the two components. And, of course, the same accumulation of scattered light and dark events had occurred for the 56 rows that had lain adjacent to the exposed strip, but on the other side away from the serial register, prior to when they were shifted into the center. Thus 56 rows on one side had received an extra frame of dark + scattered light events prior to being exposed, and 56 rows on the other had received an extra frame of dark plus scattered light events after being exposed and before being finally read out. Thus, there were 16 rows in the center that had received only one frame time of dark plus scattered light events. Voilà, a low-intensity band! Adequate masking close to the surface of the CCD with masks that matched the projected slit in size and location would have mostly eliminated this problem. However, this is not easy with a fiber-optically coupled CCD! These additional background effects greatly complicated the reduction, because they were not quantitatively measurable.

Our photometric calibration proceeded as follows. First, an appropriate dark event exposure was subtracted from all the frames (an average of many, in fact, to minimize the addition of noise). Second, we made a first-order correction for the scattered light by fitting a smoothed dark exposure to those regions which were not directly exposed, and substracting it from the whole image. The intensity of the scattered light was assumed to be constant along the slit, and to also have the same distribution perpendicular to the slit as the dark events, which, while only approximately true, appeared to be an adequate assumption for these data, given the other problems that also arose. The low intensity band in the flat fields was cor- 
rected for in a different way. We measured the average value at the edges of the band, and subtracted smooth dark (三scattered light) frames to bring the difference to zero. The contribution from the scattered light was found to be $10 \%$ ! It was found also that the flat fields varied significantly along the slit. This could not be attributed to saturation effects or incorrect subtraction of scattered light, and thus reflected real changes in the two-dimensional response of the detector during the run. We address this further below. The large-scale response variations were removed from the flat field frames in the same way as described for the ICCD data, and the resulting small-scale (pixel-to-pixel) response frame was divided into all the data.

Again, as for the ICCD, this first phase of the photometric rectification was followed by removal of the geometric distortion. Relative shifts and tilts of the images were determined from tracings of the $5577 \AA$ [O I] night sky line on the object frames and the $5015 \AA \mathrm{He}$ I line on the calibration frames. While the tilts of the lines were found to be stable, the positions of the lines were sometimes found to change by up to 2 pixels during the exposure, by comparison of the "before" and "after" arc spectra. The arc line calibration exposures were grouped into sets of three consecutive integrations having similar shifts and tilts, and summed. It proved essential to average these calibration frames because of the low $\mathrm{S} / \mathrm{N}$ ratio of the individual frames. A wavelength solution was established from mapping 34 emission lines on each of the summed exposures. The distortions were large at the ends of the spectrum, with curvature of up to 15 pixels for the lines at high and low wavelengths. Again, a two-dimensional polynomial was fitted to give a wavelength solution. The RMS residual from the fit was about 0.3 pixel, corresponding to $20 \mathrm{~km} \mathrm{~s}^{-1}$. Some large residuals were noted that were due to the extreme distortion at the ends, and to glitches, i.e., abrupt changes in wavelength along the slit. The glitches probably originated in the reducing fiber bundle feeding the CCD in the $2 \mathrm{D}$-Frutti detector chain. Glitches up to 0.5 pixel were found. Even with these problems, the averaging that results from the distribution of absorption lines along the spectrum gave velocities that were reliable to $5-10 \mathrm{~km} \mathrm{~s}^{-1}$ (see below). The distortion in the spatial direction was determined from exposures with a multihole decker aligned above the slit, that gave a series of narrow stellar-like spectra that were (roughly) evenly spaced along the slit when illuminated with the flat-field continuum source. The spectra from the holes were traced in the wavelength direction, and a two-dimensional function was fitted. Finally, the two geometric mappings were combined and inverted, and the images were rectified and rebinned logarithmically in wavelength.

The last step of the photometric calibration was then performed. The low spatial frequency structure had to be measured and removed, as in the ICCD, from the blank sky field spectra. It was found that the response function varied significantly during the run. The changes could not be characterized as a one-dimensional function such as would be expected if they were due to variations in the function describing the slit throughput, i.e., from changes in the parallelism of the slit jaws, or dust on the slit, for example. They were truly twodimensional! Similar changes were found in the intensities of the $5015 \AA \mathrm{He}$ I line in the arc spectra, and the $5577 \AA$ sky line, so these effects were real, and not due to problems with scattered light, for example. The changes were not random, but appeared to vary smoothly and periodically through the run. Since we had no other information to monitor the changes, we determined, separately for each frame, the response function which appeared to most effectively remove the large-scale variations. This was possible because the response functions were asymmetrical with respect to the center of the slit. We constructed four different large-scale response functions from four sets of sky exposures that were representative of the variations. Each step represented a change of about $10 \%$ in the large-scale response at one end of the slit. We applied all four functions to an average of the central 100 rows of each spectrum. The response function which gave the most symmetrical galaxy profile along the slit was then chosen numerically and checked visually. This uncertainty in the response function was the limiting factor in the accuracy with which the background sky could be established and removed.

Finally, the most appropriate response function was divided into each spectrum, and the sky spectrum from the ends of the slit was measured and subtracted. Since the uncertainty of the response function was highest at the ends of the slit, we ignored the outermost $20 \%$ at each end of the slit in the determination of the sky spectrum. By simulation of the possible errors caused by choosing the wrong response function, we found that the errors in the sky subtraction probably do not exceed $10 \%$.

\section{iii) RCA CCD Data}

The calibration of the ESO "barefoot" RCA CCD data was straightforward, and quite pleasurable by comparison. The approach followed mirrored that of the ICCD data. The only difference was that the cosmic-ray events were abundant on the long exposures, and so a special algorithm was written to locate the events. For each section of 100 rows of the spectrum an average profile of the galaxy intensity along the slit was calculated, and this profile was fitted to each row separately, and subtracted. The resulting difference frame with zero mean showed all the cosmic-ray events, plus systematic slanting noise patterns over the CCD. These patterns were also found in the dark frames and were probably due to noise pickup (50 $\mathrm{Hz}$ ?-ground loops?). For the cosmic-ray removal, pixels that deviated by more than $5 \sigma$ were marked, together with the surrounding pixels that deviated by more than $3 \sigma$ from zero. The number of events located in this way was proportional to the integration time, as would be expected for cosmic-ray events. The exact cutoff level was determined by eye after displaying the image. Finally, the marked pixels in the image were replaced by the value given by the fit of the first step.

The wavelength calibration was very accurate for these systems, due to the high $\mathrm{S} / \mathrm{N}$ of the arc line spectra, the low distortion and to the good charge transfer efficiency of the CCDs. The RMS residual from the fits was $5 \mathrm{~km} \mathrm{~s}^{-1}$. The slit function was very stable, and sky subtraction was straightforward. The major limiting factor was the very high readout noise of the CCD.

\section{b) Radial Velocities and Velocity Dispersions}

A tradition of more than a decade exists for the numerical determination of radial velocities and velocity dispersions of galaxies. The Fourier quotient method pioneered by Sargent $e t$ al. (1977) is still frequently used. Here we use a similar, but not identical method which we call the Fourier Fitting method. We minimize the residuals of the fitted stellar spectrum to the galaxy spectrum

$$
\chi^{2}=\sum_{i=1}^{N}\left(G_{i}-B_{i} \circ S_{i}\right)^{2}
$$


where $G$ is the galaxy spectrum, $B$ is the Gaussian broadening function, $S$ is the stellar spectrum, and o denotes convolution. The subscript $i$ denotes the measured value at an individual pixel. $\chi^{2}$ is proportional to $\tilde{\chi}^{2}$, which is the residual in the Fourier domain, given by

$$
\tilde{\chi}^{2}=\sum_{j=0}^{N-1}\left(\tilde{G}_{j}^{*}-\tilde{B}_{j}^{*} \tilde{S}_{j}^{*}\right)\left(\tilde{G}_{j}-\tilde{B}_{j} \tilde{S}_{j}\right) .
$$

The Fourier transform of a function $F$ is denoted by $\tilde{F} . \tilde{F}^{*}$ is the complex conjugate of $\tilde{F}$. The Fourier domain is the appropriate domain for the numerical fitting because the convolution is now a multiplication.

The advantage of fitting the spectra to each other instead of fitting the broadening function to the quotient of $\widetilde{G} / \widetilde{S}$ is that the error analysis is more straightforward. Errors in the quotient have a non-Gaussian distribution, thereby complicating the error analysis. If the noise in the stellar spectrum is negligible, then both methods are comparable.

Many authors have noted that the formal error estimates of the Fourier quotient method were too large, by a factor of about 1.5 (e.g., Davies and Illingworth 1983; Heckman et al. 1985; Davies and Birkinshaw 1988). We found that this discrepancy was due to an error in the determination of the formal errors. In Appendix A we derive the formal errors from a linearized least-squares fit. The error estimate derived in the Appendix was checked by numerical tests and was found to agree with the real scatter in the fitting parameters. In the Fourier quotient algorithm available at KPNO the calculated errors were a factor of $2^{1 / 2}$ too high. We believe that this discrepancy caused the overestimation of the errors that was noted by several authors. Another concern was that systematic changes in the best fitting dispersion value occur as a function of $\mathrm{S} / \mathrm{N}$ ratio. We note that other determinations of dispersions suffer from similar effects.

For example, consider the measurement of the RMS deviation from the mean of a finite number of data points. The measured values are denoted by $m_{i}$. We define $\langle m\rangle$ and $\left\langle m^{2}\right\rangle$ as the mean and mean squared measured values. If we estimate the real dispersion $\sigma^{2}$ with the estimator $\hat{\sigma}^{2}$, with

$$
\hat{\sigma}^{2}=\left\langle m^{2}\right\rangle-\langle m\rangle^{2}
$$

then $\sigma^{2}$ will be systematically too low by $\sigma^{2} / N$, where $N$ is the total number of data points. This deviation is equal to the square of the expected error of $\langle m\rangle$.

The Fourier fitting method suffers from a similar effect. The systematic error in $\sigma^{2}$ is on the order of the squared formal error of the radial velocity. Since the correction is quadratic, it is only $1 \%$ for typical dispersions near $200 \mathrm{~km} \mathrm{~s}^{-1}$ and velocity errors of $20 \mathrm{~km} \mathrm{~s}^{-1}$. It is only at quite low $\mathrm{S} / \mathrm{N}$ that this problem becomes significant. We did not attempt to correct for these systematic errors, but rather we made sure that all the spectra for which the dispersion was determined had high enough $\mathrm{S} / \mathrm{N}$.

Another widely used method to determine radial velocities is the cross-correlation method, described by Tonry and Davis (1979). We can use their method in a way that is equivalent to the Fourier fitting method defined in this paper if we take the broadening of the function $B$ to be constant and allow only the radial velocity to be changed. The advantage of this restricted mode of the Fourier fitting program is that the $\mathrm{S} / \mathrm{N}$ required to give a radial velocity is lower than the $S / N$ required to give a velocity dispersion. Hence more independent radial velocities can be generated with the restricted Fourier method.
To assure that the program fitted spectra with the desired $\mathrm{S} / \mathrm{N}$ ratio, the program summed spectra along the slit until a specified $S / N$ ratio was achieved. Afterward, the fit was performed. The input parameter which controlled the summation was the $\mathrm{S} / \mathrm{N}$ of the summed spectrum. The $\mathrm{S} / \mathrm{N}$ was not assumed to be just the square root of the number of photons in the input spectra, but was corrected for the contribution of the readout noise and the noise from the subtracted sky spectrum. The $\mathbf{S} / \mathrm{N}$ cutoffs are expressed in terms of the equivalent total number of photons that would give the same $S / N$ as in the actual summed spectrum when shot or photon noise was the only source. Typical minimum values are $3 \times 10^{5}$ photons for a determination of the velocity dispersion, and $5 \times 10^{4}$ photons for a determination of the radial velocity in the "restricted" mode. In general, these values depend on the resolution of the spectrograph and the wavelength region used. An additional check on the fitting procedure was to monitor the height of the cross-correlation peak with respect to the next largest peak in the absolute value of the cross-correlation function; this was used to establish whether the program had chosen the right peak.

Before the actual fit was done, the continuum was removed from the spectra. First, a low-order (typically fifth order) polynomial fit was subtracted from each spectrum. Second, the remaining low wavenumbers were filtered out of the Fourier transformed spectrum. The filter was an inverted cosine bell, i.e., zero from Fourier wavenumber zero to some user-defined wavenumber $\kappa\left(\approx 70 \AA^{-1}\right)$, and then smoothly rising to 1 at $2 \kappa$. Such a smooth filter is more appropriate than the Heaviside functions used in the past, although the results were not found to depend critically on this filtering.

Because of the uncertainty in the sky subtraction for the CTIO data and the KPNO data, we did not fit velocities and dispersions at radii at which the galaxy intensity was lower than a certain fraction of the sky intensity. For the CTIO data, we put this fraction at $100 \%$, and for the KPNO data at $40 \%$. These cutoffs were chosen such that the error in sky subtraction did not exceed $10 \%$ of the galaxy intensity.

The wavelength intervals used for the fit could be chosen freely. The current fast Fourier transforms implemented in the standard libraries allow spectrum lengths different from powers of two. The best wavelength intervals for the fits were determined by trial and error. The $2 \mathrm{D}$-Frutti spectra were divided into two parts, which were fitted to the stellar spectrum separately. Thus, more independent velocities were obtained in the central parts of the galaxies. A wavelength interval of 3960 $4580 \AA$ was used for the ICCD data. The $H$ and $K$ lines were not included because these features are so wide that they contribute very little to the velocity or velocity dispersion measurement. Since the galaxy-to-sky ratio is largest at longer wavelengths in these spectra, we used the part of the spectrum to the red of the $K$ line. Almost the full ESO spectra were used for the fit, since their spectral range was small. Only the part around and to the red of the $5577 \AA$ skyline was excluded.

The template stars taken by the CTIO 2D-Frutti were shifted to one velocity, and added together to form one high $\mathrm{S} / \mathrm{N}$ spectrum. We used the star HD 52071 as a template for the KPNO ICCD data, and for the ESO CCD data.

The velocity dispersions and radial velocities were determined with the full Fourier fitting program. From these results, we estimated approximate values for the velocity dispersions in the outer region of each galaxy. Then the fit was repeated with the velocity dispersion fixed to this value, to give optimum results for the radial velocity determination. 
The last step is the averaging of the data points. First, the relative velocity shifts of all individual spectra were determined from the averaged sky spectrum. Then the radial velocities from the Fourier fitting program were shifted to the same system, and the radial velocities of all spectra taken at one position angle were averaged. The formal errors from the fit were used as weights. Finally, the systemic velocity of the galaxy was determined from the major and minor axis velocities, and this value was subtracted. Hence the major and minor axis velocities have the same zero point. This procedure worked well in most cases. For the few cases where there were obvious systematic velocity differences between the two axes (large asymmetries in the folded velocity curves), we applied corrections. We know from tests and from experience, that the systemic velocity is determined much more poorly than the rotation curves, as would be expected.

The averaging allowed us to check the formal errors of the radial velocities. The RMS spread of the data points in one bin was calculated. In the inner parts, the formal error estimates for the velocities were too low, because the dispersion used for the fit was purposely kept low, being set at the dispersion of the outer parts. To correct for this effect, all errors for the radial velocities were multiplied by the ratio $\sigma(r) / \sigma($ fit $)$, where $\sigma(r)$ is the actual velocity dispersion at the radius of the measurement, and $\sigma(\mathrm{fit})$ is the value used for the cross-correlation method. In the outer parts, it was found that the error estimates from the averaging were typically between a factor of 1 and 1.4 higher than the formal error estimates. Another error estimate can be made from the asymmetry of the radial velocity profile with respect to the zero point. This method was used by Davies and Birkinshaw (1988). With this method, we found errors typically a factor of 1 to 2 lower than our formal errors. Given the systematic difference between these two alternative methods, we decided to keep to our formal error estimates for the outer parts. This gives an indication of the level of uncertainty in our error estimates, namely $30 \%-40 \%$.

\section{RESULTS}

\section{a) General Remarks}

The rotation curves and velocity dispersion profiles are plotted in Figure 1 for our program galaxies. The radial velocities and velocity dispersions have been folded about the center. The radial velocities were derived with the "restricted" Fourier fitting approach, in which the velocity dispersion was kept constant, typically equal to the velocity dispersion of the galaxy at large radii, but never higher than $200 \mathrm{~km} \mathrm{~s}^{-1}$. The actual dispersion used is not critical; as we noted earlier, the radial range over which we could determine velocities with this approach was increased, without introducing any systematic error. The error bars were derived from the formal error estimates of the Fourier fitting program. The kinematical data are tabulated in Table 7 in Appendix B. The velocity profiles of some of the galaxies observed at CTIO are not symmetric near the center. The most notable examples are NGC 1399 and NGC 1549. The centers of these galaxies saturated the 2DFrutti, and we believe that this effect is caused by the saturation, giving similar deviations as the beam-bending which occurred in older generation instruments (e.g., Kormendy and Illingworth 1982). The symmetry of the radial velocities of NGC 1549 measured by Rampazzo (1988) strengthens our conclusion that the effect is instrumental.

It is striking that of the 22 galaxies, all but one show signifi- cant rotation, even though many round galaxies were included in our sample. The one galaxy that shows no rotation, $v_{r, \max }<$ $8 \mathrm{~km} \mathrm{~s}^{-1}$ on the major axis, and even less on the minor axis, is the $\epsilon=0.05$ elliptical NGC 5846. In six cases, we find significant minor axis rotation (at the $\approx 3 \sigma$ level or greater). These galaxies are NGC 636, 1407, 1549, 4406, 7145, and 7507. For NGC 4406 and NGC 7507, the minor axis rotation exceeds the major axis rotation, while for both NGC 1549 and NGC 7145 $v_{\min } \approx v_{\mathrm{maj}}$. Thus our sample includes galaxies similar to the minor axis rotator NGC 4261, found by Davies and Birkinshaw (1986). NGC 4261 is obviously not unique. It appears, therefore, that a substantial fraction (10\%?) of ellipticals may have $v_{\text {min }}>v_{\text {maj }}$.

The discovery by Franx and Illingworth (1988) of an elliptical IC 1459 whose nucleus has a kinematically distinct, rapidly rotating component and the evidence that such components are not uncommon (Jedrzejewski and Schechter 1988; Bender 1988) has led us to scrutinize all our kinematical data for such components. A particularly interesting feature associated with these components is that they can have an angular momentum vector that is oriented quite differently from the angular momentum of the main body of the galaxy. While IC 1459 , with its counterrotating core is the most extreme case to date, we have found another galaxy, NGC 4406, that is as striking an example of this phenomena as IC 1459, as well as indications of the existence of distinct cores in four other galaxies, namely NGC 1395, 1439, 1700, and 4472. However, the results for these latter galaxies are not as significant as one would like and need confirmation. The rotational velocity in NGC 1395 has a local minimum at $\approx 7^{\prime \prime}$ on the major axis. This might be a case of a kinematical component in the core which has its angular momentum parallel to that of the outer parts. The data indicates that NGC 1439, like IC 1459, also has a counterrotating core. We will discuss these galaxies in more detail in a future paper on such cores (Franx and Illingworth 1989). However, there are two points that are of interest at this stage.

First, with regard to IC 1459, it should be noted that the rotational velocities shown here in the inner regions are lower than the central velocities given by Franx and Illingworth (1988). This is due to the asymmetries in the line profile that were described in that paper. The velocities reported here were determined by fitting a broadened star to the galaxy spectrum. Thus the line profiles were "smoothed," and the resulting velocity amplitude lowered. This is an excellent example of the difficulty of establishing the true velocities in multi-component systems (see, e.g., Illingworth and Franx 1989).

Second, these data show that NGC 4406 is particularly interesting. The $1 \sigma$ upper limit to the rotation on the major axis is $6 \mathrm{~km} \mathrm{~s}^{-1}$ for $r>5^{\prime \prime}$, yet on the minor axis we measure a quite significant $39 \pm 8 \mathrm{~km} \mathrm{~s}^{-1}$ of rotation. Yet it is not only the minor axis rotation that makes this galaxy interesting. There is also a rapidly rotating component in the core of NGC 4406 with a peak velocity $>50 \mathrm{~km} \mathrm{~s}^{-1}$ on the major axis. Thus, the projected angular momentum of the core is perpendicular to that of the outer part of the galaxy. Thus the kinematically decoupled core of IC 1459 is not a unique phenomenon.

\section{b) Average Rotational Velocities and Velocity Dispersions}

The overall goal of this program is to constrain the shapes of ellipticals by comparing the observed minor and major axis rotational velocities with those from suitably projected models. For this we would ideally like to define a quantitative pro- 

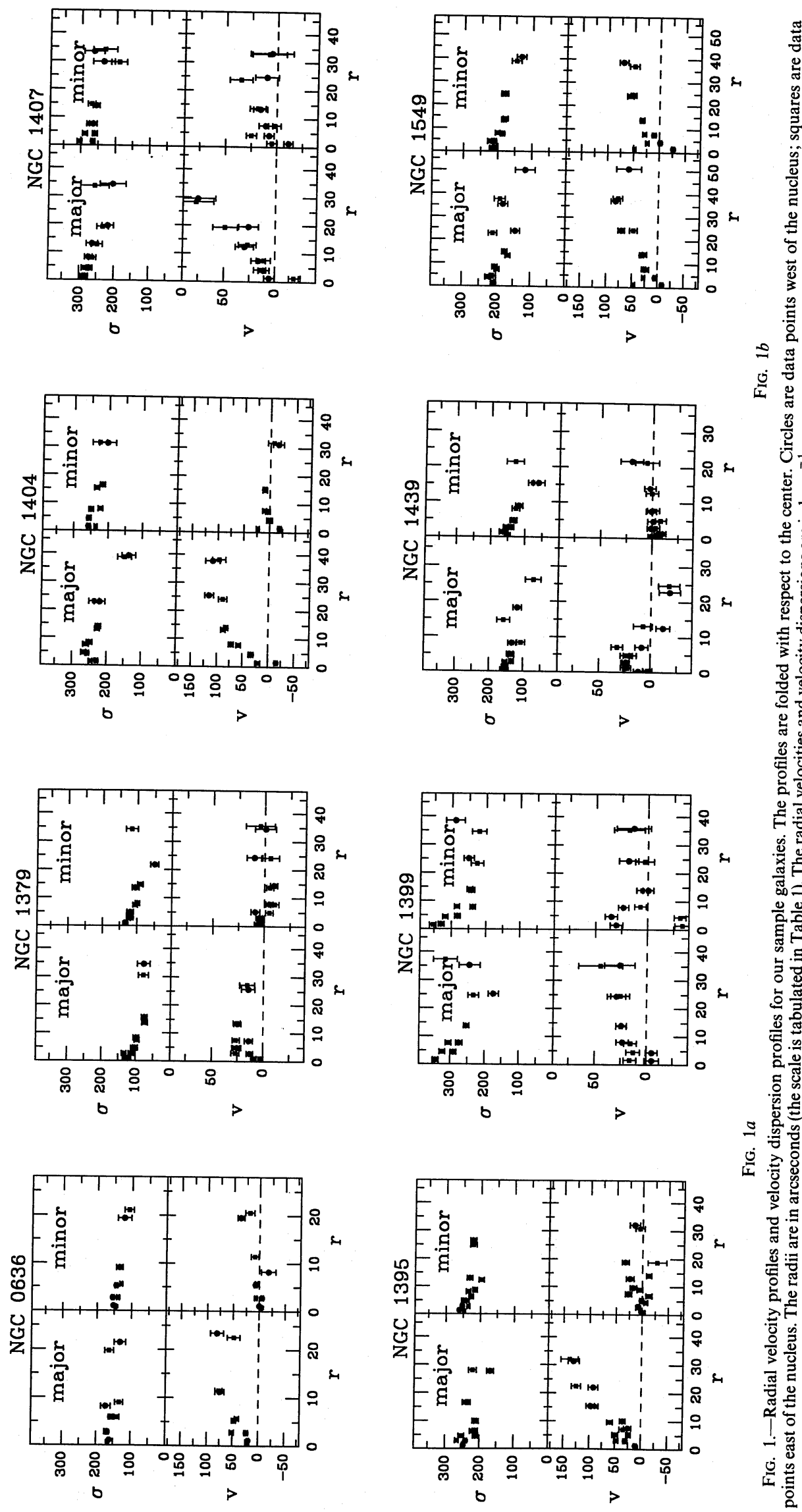

621 

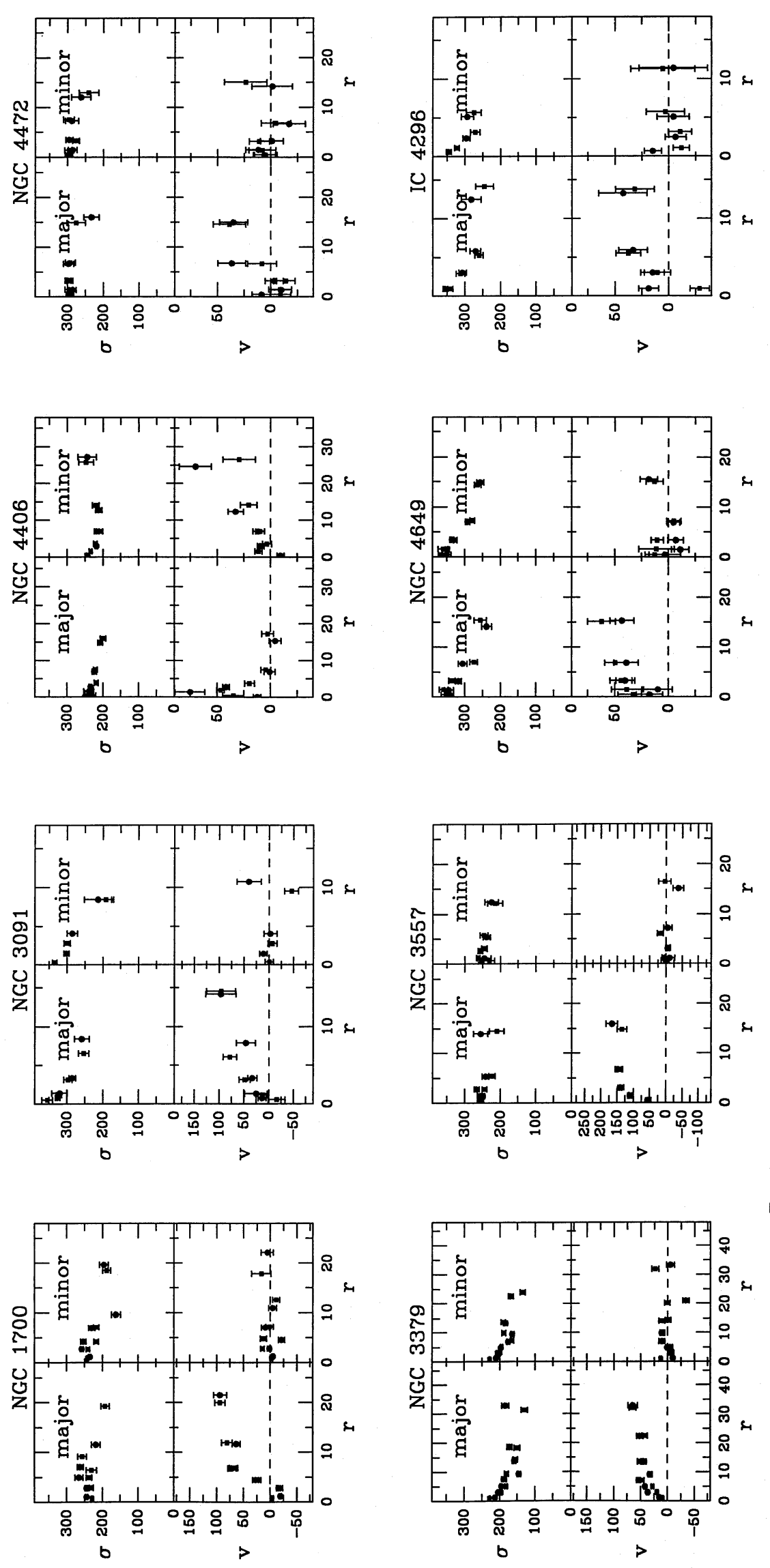

622 


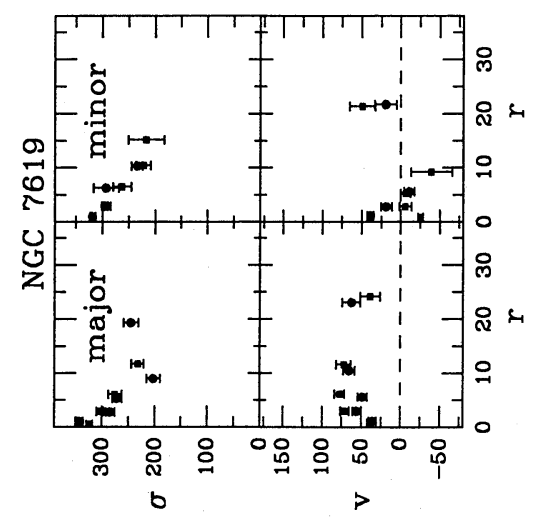

$\overrightarrow{\dot{j}}$
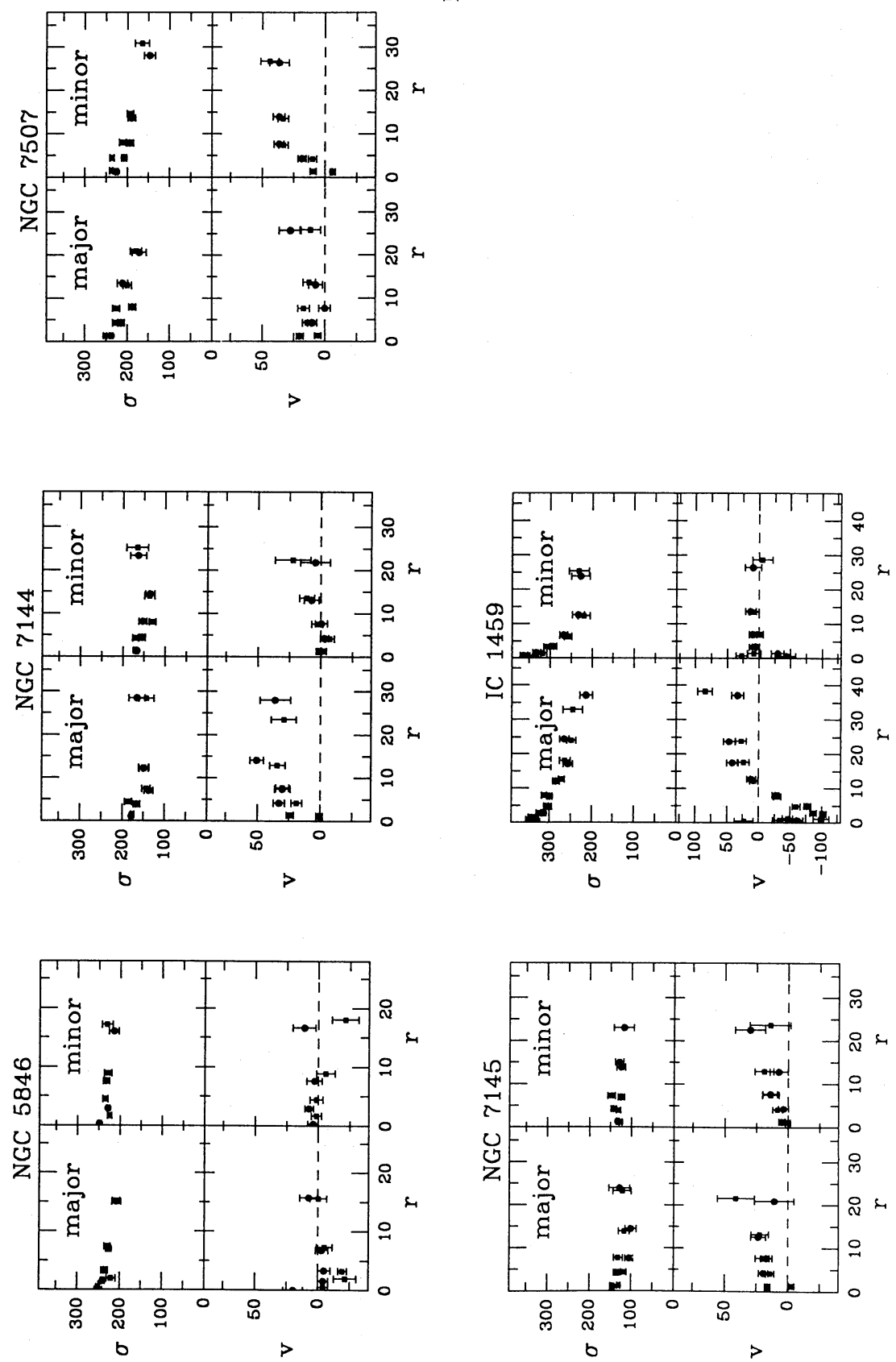

$\stackrel{9}{\dot{5}}$ 
cedure for deriving mean velocities that is independent of both distance and galaxy type. However, as with all data, ours is of uneven quality and so it was not possible to define a metric radius range over which the mean rotational velocities could be determined. We have endeavoured to minimize any subjective content by estimating the rotational velocity along each axis in two ways. First, we determined the mean rotation outside a certain radius along each axis. The inner radius of the interval used was chosen to exclude any obvious contribution from a kinematically distinct core and set to correspond to the point where the rotation curve began to level off, typically between $3^{\prime \prime}$ and $10^{\prime \prime}$. In those cases where the velocity curve is linear with radius we fitted a straight line to the observations and determined the velocity at 20", except for NGC 3091 where the data did not extend to $20^{\prime \prime}$.

Second, we fitted the simple model

$$
v=v_{0} \frac{r}{r_{v}+|r|}
$$

and from the best model fit the velocity at $20^{\prime \prime}$ was determined. The data points at $r<5^{\prime \prime}$ were not used for the fit, to avoid a contribution from the core.

The velocities determined by both methods are listed in Table 4. For the first method, where we determined the mean rotation velocity, we used the formal errors as weights. The formal errors were, however, very low for many of the galaxies observed at CTIO and KPNO (smaller than $5 \mathrm{~km} \mathrm{~s}^{-1}$ ). Fortunately, since we had multiple spectra for most of these galaxies, the formal errors could be independently checked by comparison of the independent rotational velocity results. For the CTIO data, we typically had two spectra on each axis, and for each spectrum we had two independent velocity profiles. We derived average rotational velocities from all independent profiles. The differences $\Delta V \equiv\left(V_{\text {rot }}-\left\langle V_{\text {rot }}\right\rangle\right) /(N-1)^{1 / 2}$ and the formal error of $\Delta V$ were determined, and these are plotted against each other in Figure $2 a$. A histogram of the values of $\Delta V$ is given in Figure $2 b$. The differences were divided by the square root of the number of independent spectra minus 1 , so that the spread in $\Delta V$ is equal to the real spread in $\left\langle V_{\text {rot }}\right\rangle$. It is clear that the spread in $\Delta V$ is larger than the formal error, and that the spread is not strongly dependent upon the formal error. Thus, it appears that systematic effects dominate when the formal errors fall below a few $\mathrm{km} \mathrm{s}^{-1}$. We have, therefore, set a lower limit of $5 \mathrm{~km} \mathrm{~s}^{-1}$ to the errror in the rotational velocity, and to the mean and fitted data as well (since systematic effects are involved). In Figures $2 c$ and $2 d$ we plot the differences and histogram of the differences derived from the multiple KPNO exposures. Again, we see that the formal error estimates are too small. We used the same lower limit of $5 \mathrm{~km}$ $\mathrm{s}^{-1}$ for the error in the rotational velocities derived from this data.

The model-fitting method gave independent error estimates, and these were also tabulated. In the model fitting, all data values outside $5^{\prime \prime}$ from the center were given equal weight. The error estimates obtained in this way are completely independent from the error estimates calculated from the formal errors of the velocities.

TABLE 4

MAJOR AND Minor Axis Rotation

\begin{tabular}{|c|c|c|c|c|c|c|c|c|}
\hline \multirow[b]{2}{*}{$\begin{array}{c}\text { GALAXY } \\
\text { (1) }\end{array}$} & \multicolumn{5}{|c|}{ AVERAGE Velocities } & \multicolumn{3}{|c|}{ VELOCITY FITS } \\
\hline & $\begin{array}{l}\Delta r \\
(2)\end{array}$ & $\begin{array}{l}\langle r\rangle \\
(3)\end{array}$ & $\left\langle v_{\text {major }}\right\rangle$ & $\left\langle v_{\text {minor }}\right\rangle$ & 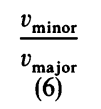 & $\begin{array}{c}v_{\text {major }} \\
(7)\end{array}$ & $\begin{array}{c}v_{\text {minor }} \\
(8)\end{array}$ & $\frac{v_{\text {minor }}}{v_{\text {major }}}$ \\
\hline NGC 636. & $8-60$ & 15.6 & $74 \pm 5$ & $20 \pm 5$ & 0.27 & $71 \pm 7$ & $21 \pm 6$ & 0.30 \\
\hline NGC 1379. & $4-60$ & 10.8 & $23 \pm 5$ & $-4 \pm 5$ & -0.17 & $20 \pm 5$ & $-1 \pm 5$ & -0.03 \\
\hline NGC $1395 \ldots \ldots \ldots$ & $5-60^{a}$ & 20 & $93 \pm 5$ & $7 \pm 5$ & 0.07 & $100 \pm 5$ & $8 \pm 5$ & 0.08 \\
\hline NGC $1399 . . . \ldots .$. & $10-60$ & 17.8 & $26 \pm 5$ & $-5 \pm 5$ & -0.21 & $27 \pm 5$ & $-11 \pm 6$ & -0.39 \\
\hline NGC $1404 \ldots$ & $10-60^{\mathrm{a}}$ & 17.5 & $90 \pm 5$ & $8 \pm 5$ & 0.09 & $90 \pm 5$ & $2 \pm 5$ & 0.02 \\
\hline NGC $1407 \ldots$. & $5-60^{a}$ & 20 & $-53 \pm 8$ & $-12 \pm 5$ & 0.23 & $-47 \pm 5$ & $-15 \pm 5$ & 0.31 \\
\hline NGC $1439 \ldots \ldots \ldots$ & $3-10$ & 6.2 & $-21 \pm 5$ & $-2 \pm 5$ & 0.11 & $-11 \pm 5$ & $0 \pm 5$ & 0.02 \\
\hline NGC $1549 \ldots \ldots \ldots$ & $5-60^{a}$ & 20 & $44 \pm 5$ & $38 \pm 5$ & 0.87 & $49 \pm 6$ & $44 \pm 6$ & 0.90 \\
\hline NGC $1700 \ldots$ & $8-60$ & 14.5 & $-80 \pm 5$ & $-4 \pm 5$ & 0.05 & $-86 \pm 12$ & $5 \pm 7$ & -0.06 \\
\hline NGC $3091 \ldots . . .$. & $5-60$ & 9.1 & $-71 \pm 10$ & $3 \pm 14$ & -0.04 & $-108 \pm 13$ & $4 \pm 16$ & -0.04 \\
\hline NGC $3379 . . . \ldots . .$. & $6-60$ & 12.9 & $-44 \pm 5$ & $-7 \pm 5$ & 0.16 & $-53 \pm 5$ & $-2 \pm 5$ & 0.03 \\
\hline NGC $3557 \ldots . . \ldots$. & $3-60$ & 5.0 & $-140 \pm 5$ & $3 \pm 5$ & -0.02 & $-153 \pm 5$ & $15 \pm 8$ & -0.10 \\
\hline NGC $4406 \ldots . . \ldots$. & $5-60^{a}$ & 20 & $1 \pm 5$ & $-39 \pm 8$ & -35 & $13 \pm 26$ & $-39 \pm 5$ & -2.92 \\
\hline NGC $4472 \ldots . . .$. & $5-60$ & 10.1 & $29 \pm 7$ & $-3 \pm 8$ & -0.12 & $42 \pm 15$ & $9 \pm 11$ & 0.22 \\
\hline NGC $4649 \ldots \ldots \ldots$ & $3-60$ & 7.6 & $-46 \pm 5$ & $3 \pm 5$ & -0.07 & $-53 \pm 5$ & $15 \pm 7$ & -0.28 \\
\hline IC $4296 \ldots \ldots \ldots \ldots$ & $5-60$ & 7.3 & $-36 \pm 8$ & $0 \pm 10$ & -0.01 & $-39 \pm 11$ & $3 \pm 10$ & -0.06 \\
\hline NGC $5846 \ldots . . . .$. & $3-60$ & 7.7 & $-7 \pm 5$ & $-1 \pm 5$ & 0.15 & $-2 \pm 8$ & $4 \pm 6$ & -1.98 \\
\hline NGC $7144 \ldots \ldots \ldots$ & $5-60$ & 11.6 & $36 \pm 5$ & $-5 \pm 5$ & -0.14 & $36 \pm 5$ & $-11 \pm 5$ & -0.31 \\
\hline NGC $7145 \ldots \ldots \ldots$ & $5-60$ & 10.8 & $21 \pm 5$ & $15 \pm 5$ & 0.72 & $26 \pm 5$ & $20 \pm 5$ & 0.74 \\
\hline IC $1459 \ldots \ldots \ldots$ & $15-60$ & 26.4 & $40 \pm 5$ & $2 \pm 10$ & 0.05 & $23 \pm 15$ & $5 \pm 8$ & 0.22 \\
\hline NGC $7507 \ldots \ldots . .$. & $5-60$ & 12.4 & $-11 \pm 5$ & $36 \pm 5$ & -3.3 & $-17 \pm 5$ & $37 \pm 5$ & -2.13 \\
\hline NGC $7619 \ldots \ldots \ldots$ & $5-60$ & 9.1 & $-63 \pm 5$ & $3 \pm 5$ & -0.05 & $-60 \pm 5$ & $-22 \pm 14$ & 0.36 \\
\hline
\end{tabular}

NOTES.-Col. (2) gives the radial interval in arcseconds over which the velocities are averaged. The error-weighted mean radius is given in col. (3), and the rotational velocities for the major and minor axes are listed in cols. (4) and (5) with their errors. Col. (6) gives the ratio of minor to major axis rotation. Cols. (7)-(9) give the results from the model fit to the radial velocities. The major axis rotation (col. [7]), minor axis rotation (col. [8]), and their ratio (col. [9]) are listed. All velocities are given in km s ${ }^{-1}$, with the sense of the rotation being defined by the sign in the eastern sector (positive radii; see Table 7).

a Linear fits have been used for both axes of NGC 1395, NGC 1407, NGC 1549, and for the minor axis of NGC 4406. 

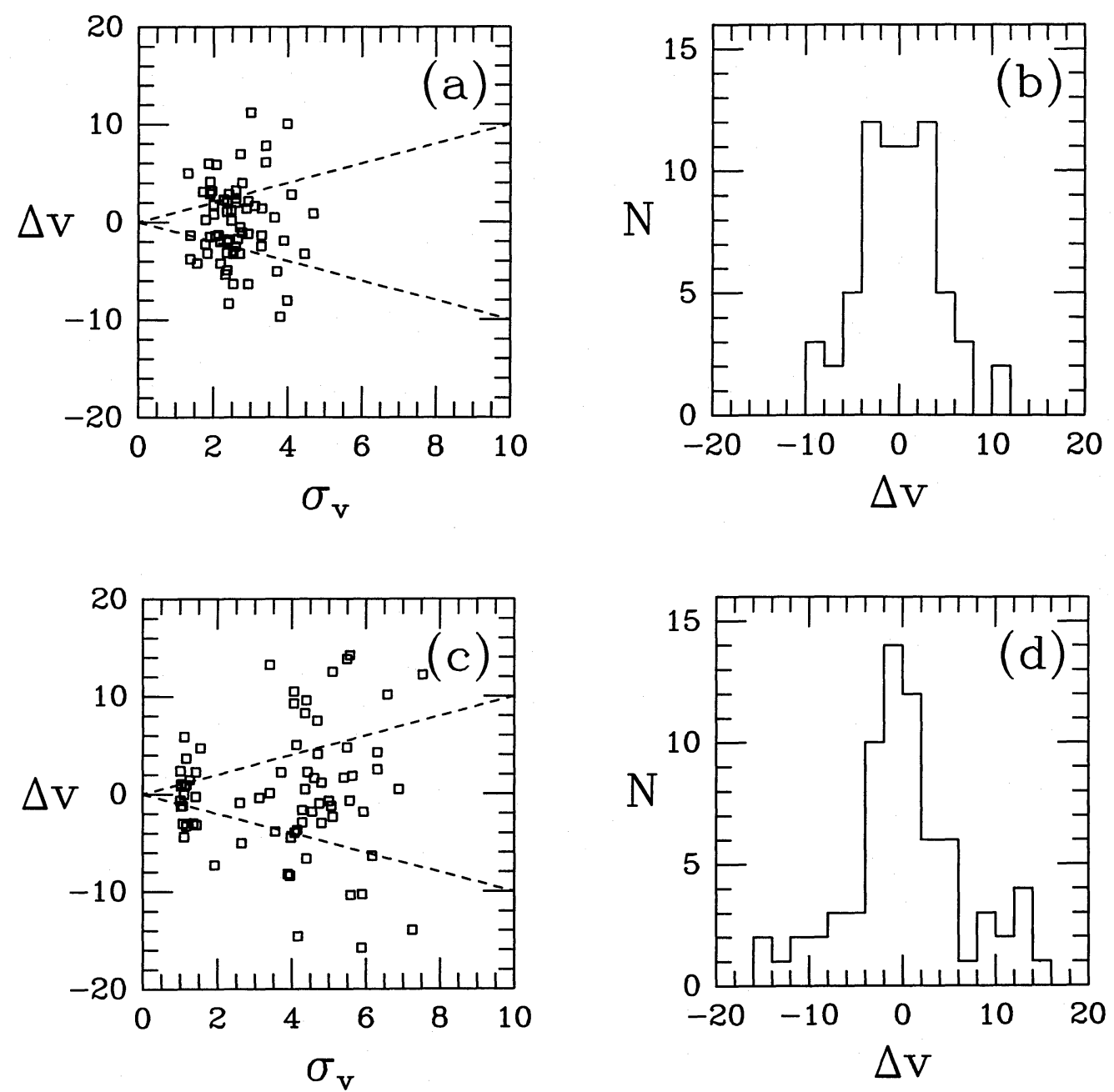

Fig. 2- The difference $\Delta V$ in the mean radial velocity between spectra taken at the same position angle plotted against the expected error for the CTIO data (a), and for the KPNO data $(c)$. The differences and errors are divided by $(N-1)^{1 / 2}$ so that the error is also a good indication of the error of the mean. $N$ is the total number of spectra at one position angle. The dotted line indicates equality, i.e., $\Delta V=\sigma_{v}$. Histograms of the errors for the CTIO and KPNO data are shown in (b) and $(d)$, respectively.

There are some data in the literature with which we can compare our results. The major axis velocities of the galaxies NGC 3379, 4472, 7619, IC 4296 are listed by Davies et al. (1983). Rampazzo (1988) presents radial velocities of NGC 1549 along the major and minor axis. Bender (1988) and Wagner, Bender, and Möllenhof (1988) give the major and minor axis velocities of NGC 4406. Davies and Birkinshaw (1988) present data along four position angles for NGC 3379 and NGC 4472. Our results agree well with these values, having a mean difference in the sense $\left\langle v_{\text {rot, us }}\right\rangle /\left\langle v_{\text {rot, others }}\right\rangle$ of $20 \%$. The most recent data agree best.

Bertola et al. (1988) have measured the minor axis rotation in NGC 3557, and note the possible detection of rotation between $10^{\prime \prime}$ and $15^{\prime \prime}$. Our higher $\mathrm{S} / \mathrm{N}$ data do not show any significant rotation along the minor axis. This may be the result of the different slit position angles used $\left(129^{\circ}\right.$ vs. $\left.122^{\circ}\right)$.

While Binney (1985) chose to use the parameter $\mu \equiv$ $v_{\text {minor }} /\left(v_{\text {minor }}^{2}+v_{\text {major }}^{2}\right)^{1 / 2}$ to characterize the degree to which ellipticals depart from the classical axisymmetric oblate figure, we have chosen to use the difference between the photometric minor axis and the kinematical axis ( $\approx$ rotational axis). The two approaches are equivalent. We have used the latter approach because the comparison of the kinematical angle and the photometric angle is more straightforward when the kinematic data do not lie exactly along the photometric axes, as is often the case.

From the ratio $v_{\text {minor }} / v_{\text {major }}$ and the position angle $\hat{\Theta}_{\text {obs, min }}$ of the observed minor axis slit position we estimate the kinematic angle $\hat{\Pi}$ by

$$
\left|\tan \left(\hat{\Pi}-\hat{\Theta}_{\mathrm{obs}, \min }\right)\right|=\left|\frac{v_{\text {minor }}}{v_{\text {major }}}\right| .
$$

The angle $\hat{\Pi}$ is chosen such that the maximum radial velocity occurs near $\hat{\Pi}-90^{\circ}$. Equation (5) is based on the assumption that the rotational velocity at constant radius is proportional to $\sin (\hat{\Pi}-\hat{\Theta})$, where $\hat{\Theta}$ is the position angle of the measured point.

For the velocity field of a disk with circular streaming or for figure rotation, the kinematic angle defined here corresponds 
TABLE 5

Kinematic ANGle

\begin{tabular}{|c|c|c|c|c|c|c|}
\hline \multirow[b]{2}{*}{$\begin{array}{l}\text { GaLAXY } \\
\text { (1) }\end{array}$} & \multicolumn{3}{|c|}{ Average Velocities } & \multicolumn{3}{|c|}{ Velocity Fits } \\
\hline & $\begin{array}{c}\hat{\Pi}-\hat{\Theta}_{\text {obs, min }} \\
\text { (2) }\end{array}$ & గ & $\begin{array}{l}\hat{\Psi} \\
(4)\end{array}$ & $\hat{\Pi}-\hat{\Theta}_{(5)}$ & $\begin{array}{l}\hat{\Pi} \\
(6)\end{array}$ & $\begin{array}{l}\dot{\Psi} \\
\text { (7) }\end{array}$ \\
\hline NGC 636. & $15 \pm 4$ & 165 & $17 \pm 4$ & $17 \pm 5$ & 167 & $25 \pm 5$ \\
\hline NGC 1379. & $-10 \pm 12$ & 87 & $-2 \pm 12$ & $-2 \pm 14$ & 95 & $1 \pm 14$ \\
\hline NGC 1395.. & $176 \pm 3$ & 179 & $-5 \pm 3$ & $176 \pm 3$ & 179 & $-5 \pm 3$ \\
\hline NGC 1399.. & $-168 \pm 11$ & 217 & $16 \pm 11$ & $-159 \pm 11$ & 226 & $27 \pm 11$ \\
\hline NGC $1404 .$. & $175 \pm 3$ & 249 & $-4 \pm 3$ & $179 \pm 3$ & 253 & $-10 \pm 3$ \\
\hline NGC $1407 .$. & $-167 \pm 6$ & 341 & $15 \pm 6$ & $-163 \pm 6$ & 345 & $19 \pm 6$ \\
\hline NGC 1439. & $-174 \pm 13$ & 314 & $11 \pm 13$ & $-179 \pm 25$ & 309 & $6 \pm 25$ \\
\hline NGC 1549. & $139 \pm 5$ & 176 & $-34 \pm 5$ & $138 \pm 5$ & 175 & $-35 \pm 5$ \\
\hline NGC $1700 .$. & $-3 \pm 4$ & 358 & $-2 \pm 4$ & $3 \pm 5$ & 4 & $5 \pm 5$ \\
\hline NGC 3091. & $2 \pm 11$ & 59 & $2 \pm 11$ & $2 \pm 8$ & 59 & $2 \pm 8$ \\
\hline NGC 3379 . & $-171 \pm 6$ & 347 & $6 \pm 6$ & $-178 \pm 5$ & 340 & $0 \pm 5$ \\
\hline NGC 3557 . & $179 \pm 2$ & 308 & $4 \pm 2$ & $174 \pm 3$ & 303 & $0 \pm 3$ \\
\hline NGC 4406. & $-92 \pm 7$ & 298 & $87 \pm 7$ & $-109 \pm 34$ & 281 & $70 \pm 34$ \\
\hline NGC 4472 . & $-173 \pm 16$ & 259 & $6 \pm 16$ & $168 \pm 15$ & 240 & $-12 \pm 15$ \\
\hline NGC 4649. & $4 \pm 6$ & 16 & $6 \pm 6$ & $15 \pm 7$ & 27 & $14 \pm 7$ \\
\hline IC $4296 \ldots$ & $180 \pm 17$ & 330 & $-1 \pm 17$ & $176 \pm 15$ & 326 & $-3 \pm 15$ \\
\hline NGC 5846. & $-172 \pm 38$ & 358 & $-3 \pm 38$ & $117 \pm 101$ & 287 & $-54 \pm 101$ \\
\hline NGC 7144. & $-8 \pm 8$ & 109 & $-4 \pm 8$ & $-17 \pm 8$ & 100 & $-10 \pm 8$ \\
\hline NGC 7145. & $144 \pm 11$ & 181 & $-35 \pm 11$ & $143 \pm 9$ & 180 & $-35 \pm 9$ \\
\hline IC $1459 \ldots$ & $3 \pm 15$ & 132 & $2 \pm 15$ & $12 \pm 21$ & 141 & $12 \pm 21$ \\
\hline NGC 7507 & $73 \pm 8$ & 88 & $76 \pm 8$ & $65 \pm 7$ & 80 & $70 \pm 7$ \\
\hline NGC 7619. & $177 \pm 5$ & 301 & $-5 \pm 5$ & $-160 \pm 12$ & 324 & $19 \pm 12$ \\
\hline
\end{tabular}

NoTES.-The observed kinematic offset angles from the average rotation velocities (col. [2]) and the model fits (col. [5]) are listed. The resulting kinematic angles are given in cols. (3) and (6). These are given with respect to north, and positive toward east. Cols. (4) and (7) give the kinematic misalignment, which is the position angle difference between the photometric minor axis and the kinematic axis. All angles are given in degrees.

exactly to the position angle of the rotational axis. Since we have measured only two position angles, we cannot test whether this assumption is correct. This uncertainty is of little consequence for the subsequent detailed comparison with triaxial models (FIZ), since we will derive the kinematic angle $\hat{\Pi}$ in the same way from the models, thereby minimizing any systematic effects.

We will characterize the galaxies by the kinematic misalignment angle defined by

$$
\hat{\Psi}=\hat{\Pi}-\hat{\Theta}_{\min },
$$

where $\widehat{\Theta}_{\min }$ is the position angle of the photometric minor axis. It was determined at the radius at which the rotational velocities were determined. This radius is the error weighted radius $\langle r\rangle$ listed in Table 4 for the kinematic misalignment calculated from the average velocities, and $20^{\prime \prime}$ for the kinematic misalignment calculated from the velocity fit. Care in the choice of this radius is important for the galaxies which show a position angle twist. Since the photometric minor axis position angle is ambiguous by $180^{\circ}$, we chose the value which was nearest to the kinematic axis. Hence the kinematic misalignment is restricted to $-90^{\circ}<\hat{\Psi} \leq 90^{\circ}$. The misalignment angles for our program galaxies are listed in Table 5 .

We have drawn a histogram of the observed values of $|\hat{\Psi}|$ in Figure 3 . We note that $60 \%$ of the program galaxies have $|\hat{\Psi}|<10^{\circ}$. This fraction may be an underestimate, since observational uncertainty in $\hat{\Pi}$ or $\hat{\Theta}_{\text {min }}$ will bias $|\hat{\Psi}|$ toward larger values for small $|\hat{\Psi}|$, i.e., for $\hat{\Psi} \rightarrow 0$. However, it is striking to note that two ellipticals have $70^{\circ} \leq|\hat{\Psi}| \leq 90^{\circ}$, like NGC 4261 (Davies and Birkinshaw 1986), and that the intermediate region is also populated.

Finally, we have derived average dispersions from the center to $r_{e} / 2$, with all data points being weighted equally. These velocity dispersions are denoted as $\sigma_{0}$ and are tabulated in Table 6. We have compared our velocity dispersions with the values listed by Davies et al. (1987). We averaged their raw data points, and took an average of our data points which fell within the aperture used by Davies et al. We find good agreement between the two sets, with an rms error of $8 \%$. Since our new sample includes a significant number of quite round ellipticals, a range in ellipticity that has not been investigated significantly in the past, we have plotted the usual $v_{\text {rot }} / \sigma_{0}$ versus $\epsilon$ diagram in Figure 4. The mean ellipticities $\epsilon$ were taken from Table 1 . We have used both the major axis velocity $v_{\text {major }}$ and

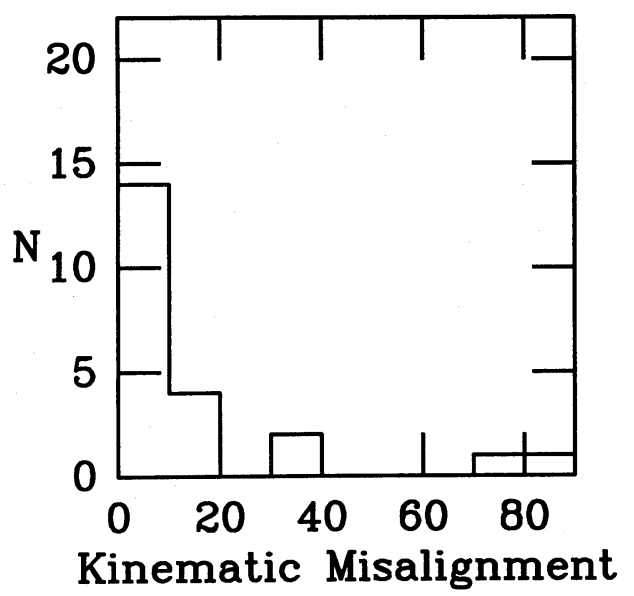

FIG. 3.-Histogram of $|\hat{\Psi}|$, the absolute value of the kinematic misalignment angle. 
TABLE 6

Average Velocity Dispersions

\begin{tabular}{|c|c|c|c|}
\hline $\begin{array}{l}\text { Galaxy } \\
\text { (1) }\end{array}$ & $\begin{array}{c}\text { Average } \\
\sigma_{0}{ }^{\mathrm{a}} \\
(2)\end{array}$ & $\begin{array}{c}\text { Major Axis Fit } \\
\alpha^{\mathbf{b}} \\
(3)\end{array}$ & $\begin{array}{c}\text { Minor Axis Fit } \\
\alpha^{\mathbf{b}} \\
(4)\end{array}$ \\
\hline NGC 636 . & $151 \pm 5$ & $-0.02 \pm 0.01$ & $-0.03 \pm 0.01$ \\
\hline NGC 1379. & $110 \pm 5$ & $-0.14 \pm 0.02$ & $-0.11 \pm 0.02$ \\
\hline NGC $1395 .$. & $234 \pm 5$ & $-0.05 \pm 0.01$ & $-0.05 \pm 0.01$ \\
\hline NGC $1399 .$. & $292 \pm 6$ & $-0.13 \pm 0.01$ & $-0.14 \pm 0.01$ \\
\hline NGC 1404 .. & $243 \pm 5$ & $-0.02 \pm 0.01$ & $-0.03 \pm 0.01$ \\
\hline NGC 1407. & $257 \pm 14$ & $-0.06 \pm 0.01$ & $-0.04 \pm 0.01$ \\
\hline NGC 1439. & $136 \pm 7$ & $-0.06 \pm 0.02$ & $-0.06 \pm 0.01$ \\
\hline NGC 1549. & $201 \pm 5$ & $-0.05 \pm 0.01$ & $-0.07 \pm 0.01$ \\
\hline NGC 1700 . & $240 \pm 5$ & $0.02 \pm 0.01$ & $-0.03 \pm 0.01$ \\
\hline NGC 3901. & $286 \pm 12$ & $-0.11 \pm 0.02$ & $-0.08 \pm 0.01$ \\
\hline NGC 3379 . & $189 \pm 3$ & $-0.11 \pm 0.01$ & $-0.09 \pm 0.01$ \\
\hline NGC 3557 . & $244 \pm 9$ & $-0.02 \pm 0.01$ & $-0.03 \pm 0.01$ \\
\hline NGC 4406 . & $226 \pm 7$ & $-0.03 \pm 0.01$ & $-0.03 \pm 0.01$ \\
\hline NGC 4472 . & $284 \pm 11$ & $-0.01 \pm 0.01$ & $-0.02 \pm 0.01$ \\
\hline NGC 4649 . & $315 \pm 8$ & $-0.08 \pm 0.01$ & $-0.09 \pm 0.01$ \\
\hline IC $4296 \ldots$ & $298 \pm 11$ & $-0.13 \pm 0.02$ & $-0.11 \pm 0.02$ \\
\hline NGC 5846 & $230 \pm 6$ & $-0.03 \pm 0.01$ & $-0.02 \pm 0.01$ \\
\hline NGC 7144. & $157 \pm 6$ & $-0.06 \pm 0.02$ & $-0.07 \pm 0.02$ \\
\hline NGC $7145 \ldots \ldots$ & $128 \pm 6$ & $-0.07 \pm 0.02$ & $0.02 \pm 0.02$ \\
\hline IC $1459 \ldots$ & $302 \pm 7$ & $-0.06 \pm 0.01$ & $-0.12 \pm 0.01$ \\
\hline NGC 7507. & $215 \pm 5$ & $-0.09 \pm 0.01$ & $-0.07 \pm 0.01$ \\
\hline NGC $7619 \ldots \ldots \ldots$ & $276 \pm 10$ & $-0.10 \pm 0.01$ & $-0.08 \pm 0.01$ \\
\hline
\end{tabular}

${ }^{a}$ Average velocity dispersion along the major and minor axis out to $r_{e} / 2$ are given in $\mathrm{km} \mathrm{s}^{-1}$. The data points are not weighted by their errors.

b Power-law index $\alpha$ from the fit using the model of eq. (6) for the major and minor axis, respectively. The formal errors from the fit are given.

the total velocity $v_{\text {tot }}=\left(v_{\text {minor }}^{2}+v_{\text {major }}{ }^{2 / 1}\right.$ for the rotational velocity $v_{\text {rot }}$. It is clear from Figure 4 that the galaxies are distributed quite homogeneously in the area under the curve of oblate isotropic rotators. There are no galaxies that show rotation that is significantly higher than what is expected from this model. This is somewhat surprising, since such cases are expected if galaxies are triaxial. However, as the rotational velocities of galaxies correlate with absolute magnitude, it is very difficult to use the $v / \sigma$ versus $\epsilon$ diagram for the analysis of intrinsic shapes.

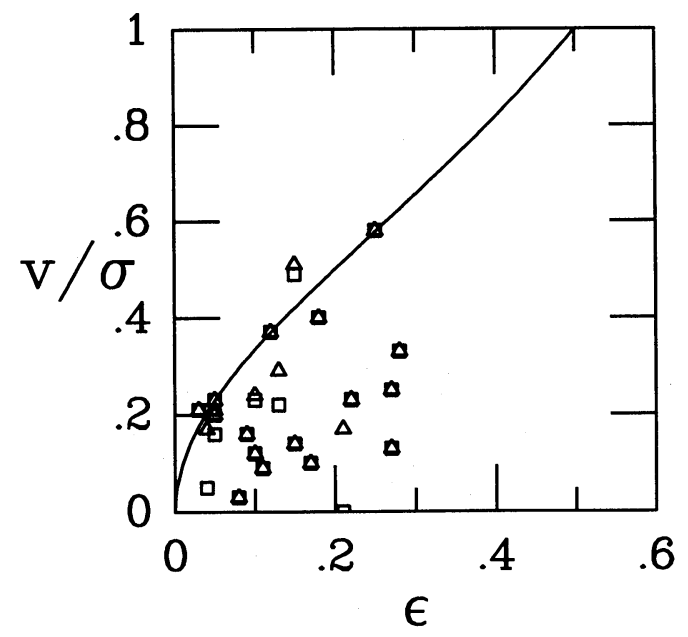

FIG. 4.-The $v_{\text {rot }} / \sigma_{0}$ vs. $\epsilon$ diagram. The squares are the values derived when $v_{\text {rot }}=v_{\text {maj }}$, and the triangles are those for which $v_{\text {rot }}=v_{\text {tot }}$. The ellipticity $\epsilon$ is the mean ellipticity listed in col. (8) of Table 1 . The line is that expected for rotating isotropic oblate galaxies (e.g., Davies et al. 1983).
We have also determined dispersion gradients by calculating the power-law index $\alpha$

$$
\sigma(r)=\sigma r^{\alpha}
$$

The resulting values are presented in Table 6 . We find a shallow gradient for most of the galaxies, with an average $\langle\alpha\rangle=-0.06$. The shallow gradient makes it very difficult to determine along which axis the dispersion falls off most rapidly. When plotted on top of each other, the major and minor axis velocity dispersion profiles are in most cases indistinguishable. Only for IC 1459 we do find a difference. The data is still not of high enough quality to determine axial ratios for contours of constant velocity dispersion. Such axial ratios would give interesting information on the internal dynamical structure of the galaxies (e.g., Statler 1987).

\section{DISCUSSION AND SUMMARY}

We have measured major and minor axis rotation with a typical uncertainty of only $5 \mathrm{~km} \mathrm{~s}^{-1}$ for 22 elliptical galaxies. All galaxies showed rotation at the $4 \sigma_{V}$ level, with one exception, NGC 5846, a round $(\langle\epsilon\rangle \approx 0.05)$ elliptical in which the $1 \sigma$ upper limit to the rotation on either axis is $v_{\text {rot }}<12 \mathrm{~km}$ $\mathrm{s}^{-1}$. Kinematical axes ("rotation axes") were derived for these galaxies. At least $60 \%$ of the galaxies studied have their kinematical axis within $10^{\circ}$ of the photometric minor axis. However, six ellipticals how significant minor axis rotation. Of these two galaxies (NGC 4406 and 7507), or $9 \%$ of the sample, show rotation on the minor axis larger than that on the major axis, two more show $v_{\text {minor }} \approx v_{\text {major }}$ (NGC 1549 and 7145), while a further two (NGC 636 and 1407) also show significant minor axis rotation, making $27 \%$ in all that show such rotation.

Minor axis rotation is expected naturally if elliptical galaxies are triaxial. Then projection effects alone can cause the misalignment of the apparent kinematical and photometric axes. The histogram of misalignment angles (Fig. 3) shows that the kinematical axes of ellipticals lie preferentially near the projected short axes of these galaxies. This implies that the direction of the intrinsic rotation axis lies preferentially near the intrinsic short axis. This is not the general case expected for galaxies with triaxial figures that have insignificant figure rotation. In that case streaming around the minor and major axis is allowed, and we might expect a random direction for the angular momentum in the plane of the major and minor axis. Our data shows that the angular momentum is not projected randomly. The direction of the angular momentum is clearly related to the shape of the galaxy, and, in particular, it is coupled to the orientation of the short axis of the figure. This relation results either from stellar dynamical constraints, or from the formation history of ellipticals. For example, figure rotation (tumbling about the short axis) could play an important role and may restrict the streaming about the long axis (e.g., de Zeeuw 1987), or the galaxies are near to oblate and have only a small fraction of phase space occupied by major axis tube orbits. Alternatively, events during the formation of ellipticals may have conspired to align the short axis of the figure and the angular momentum.

Our data strengthen Binney's (1985) conclusions that ellipticals that rotate about their long axes ("spindle"-like) are rare, and that it is quite unlikely that ellipticals are generically prolate tumbling bars. We find four galaxies, i.e., $18 \%$, of our galaxies which have a kinematic misalignment higher than $18^{\circ}$, corresponding to Binney's minor axis rotation parameter 
$\mu=0.3$, where $\mu=v_{\text {minor }} /\left(v_{\text {minor }}^{2}+v_{\text {major }}^{2}\right)^{1 / 2}$. Binney introduced the "triaxiality" parameter $Z$, which is zero for oblate models and one for prolate models. Very roughly, the measured fraction with $\mu \geq 0.3$ is between the fractions predicted by Binney's models with $Z=0.2$ (near to oblate) and $Z=0.5$ ("optimally triaxial"). We investigate this more quantitatively in a later paper (FIZ).

We cannot conclude that the galaxies without minor axis rotation are oblate. This is because triaxial galaxies show little minor axis rotation for many viewing angles. Hence there is a large probability of finding a small misalignment if galaxies rotate intrinsically around their shortest axis. This is true for galaxies which are "optimally triaxial," but also for galaxies near to prolate. FIZ discuss this in detail and give probability distributions. In a similar way it is impossible to draw definite conclusions about the galaxies which show relatively low major axis rotation (with respect to the minor axis rotation). They may be triaxial and rotating around their shortest axis, or triaxial-prolate and rotating around their longest axis. If prolate galaxies of the latter type exist, they have to be less frequent than 1 in 10 , because none of them would show major axis rotation.

As isophote twists are thought to be produced by changes in the intrinsic flattening of triaxial galaxies, it seems interesting to see whether there is a correlation between isophote twist and minor axis rotation. The isophotes twists are the logarithmic gradients in major axis position angle $\Delta \Theta_{\text {major }} / \Delta \log r$ at the radius of the kinematic observations listed in column (3) of Table 4. In Figure 5 the kinematic misalignments are plotted against isophote twist. In Figure $5 a$ the twist and the misalignment are calculated anticlockwise from the north. In Figure $5 b$ we have folded the misalignment angles and the isophote twist around zero, in such a way that only positive misalignments occur. The points which had negative misalignment were rotated by $180^{\circ}$ with respect to the origin, so both the misalignment and the twist changed sign. The plot is remarkable in two aspects: First, the galaxies with very high kinematic misalignment $\left(>60^{\circ}\right)$ show a small photometric twist, while the galaxies with intermediate misalignments all show large photometric twists. Second, the galaxies with large photometric twist all have a twist of the sign opposite to the sign of the misalignment. The only exception is NGC 7145, which is a very round galaxy, and for which the position angle twist is difficult to determine at the small radius of $12^{\prime \prime}$. The first effect may be thought to be due to a slight difference in the scale at which we calculate the position angle and the kinematic axis. It may also be due to projection effects: if a triaxial galaxy has axial ratios which change with radius, then we expect to see the largest apparent position angle twist at those viewing angles where we also see relatively strong major and minor axis rotation (e.g., FIZ). However, the second effect implies that the minor axis at small radii lies nearer to the kinematic axis then the minor axis at the radius where we measured the kinematic axis! In Figure 6 we have drawn the minor axis position angles of NGC 636, NGC 1549, and NGC 7145, which are the best examples, and the kinematic angles are indicated. It is very surprising to see that the position angle difference between the kinematic axis and the position angle becomes larger with radius for $r>10^{\prime \prime}$ for all galaxies. The position angle for NGC 7145 is uncertain in the center, because it is very round $(\epsilon=0.05)$, and is influenced by seeing to large radii (see Paper I). We do not have a large enough sample yet to establish whether the correlation between twist and misalignment is accidental or not. If it turns out to be common to twisting galaxies, then it has the interesting consequence that the intrinsic shapes of the galaxies may be different in the inner regions that in the outer regions. Specifically it might imply that galaxies are nearer to oblate at the inner regions, and more triaxial in the outer regions. We note that such an effect has been predicted already by Norman, May, and van Albada (1985), and Gerhard (1987), who found that scattering by a supermassive black hole destroys the triaxiality near the centers of the galaxies in a Hubble time.

We searched for other correlations between the kinematics and properties in the optical, radio, and X-ray wavelength domain. A complicating factor is that many quantities correlate with optical magnitude. Our sample is too small to separate such effects from any other correlations.

We have found kinematically distinct nuclear components in $10 \%-20 \%$ of our galaxies. The real fraction may well be significantly higher, since such nuclear components will not be recognized if their angular momenta are directed unfavorably with respect to the observer (i.e., along the line of sight). Furthermore, such components can also be difficult to detect when their angular momenta are aligned with those of the outer regions (cf. NGC 1395). Since it appears also that these cores do not have an obvious photometric signature (cf. IC 1459:
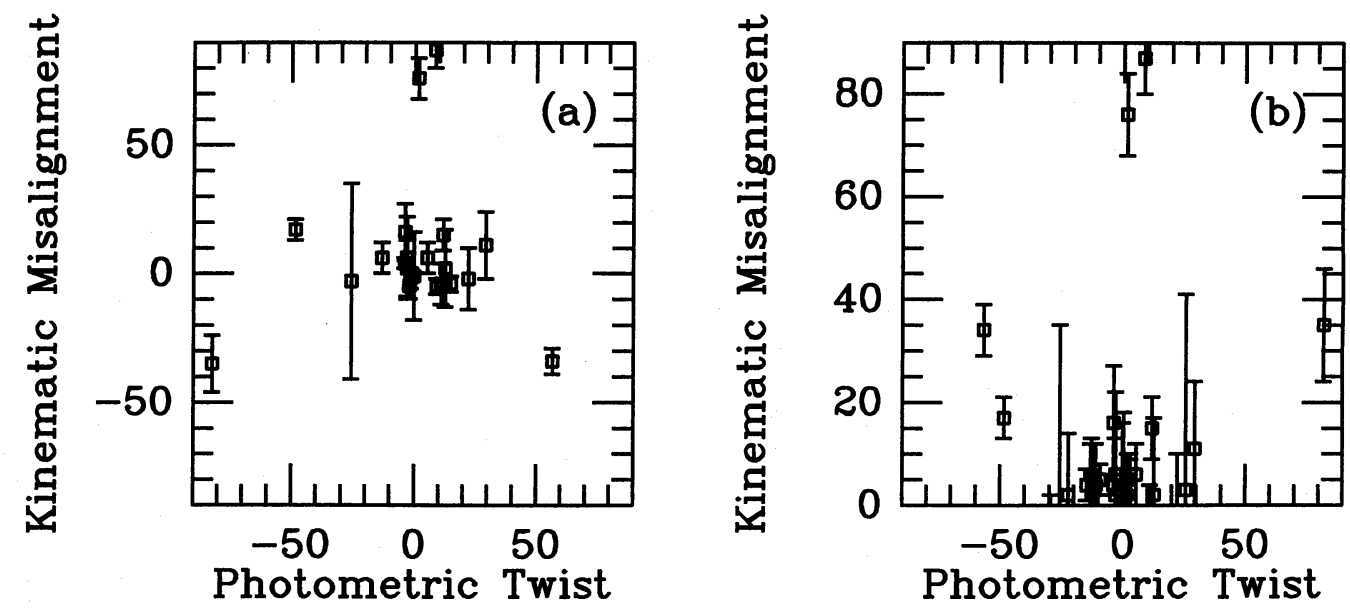

FIG. 5. - The kinematic misalignment angle plotted vs. the photometric twist, taken from Paper I and the literature. In (a) the angles are all measured from north through east, whereas in $(b)$ the points are folded with respect to the center so that the misalignments are positive. 

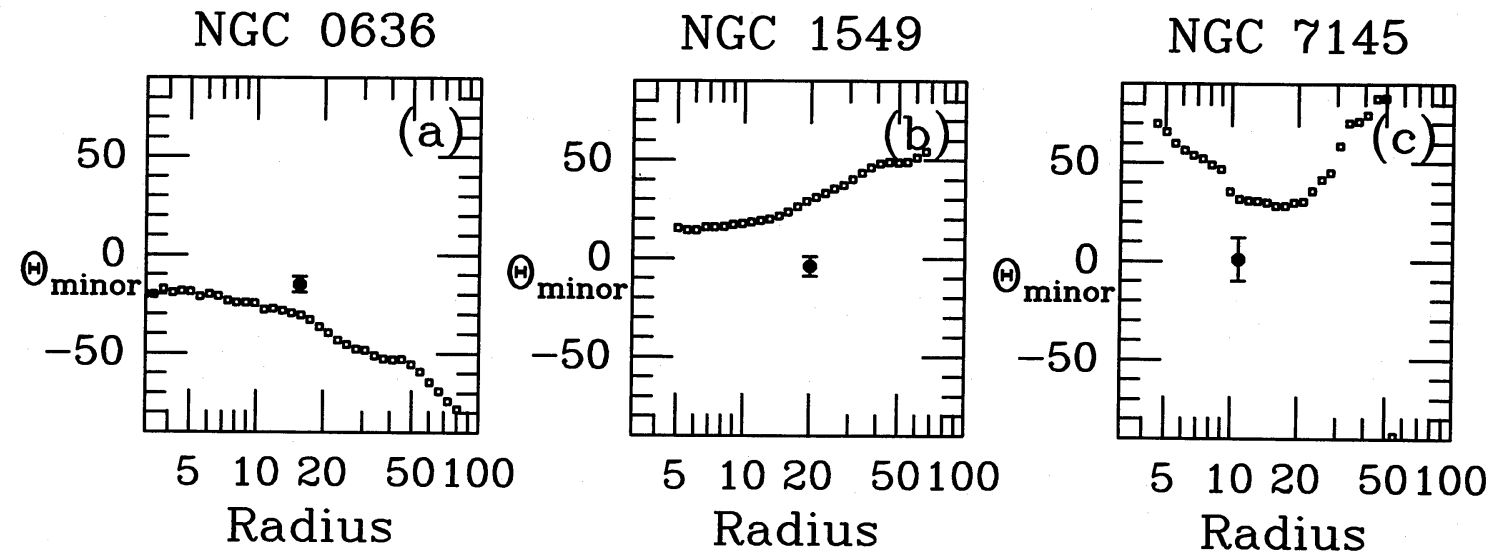

FIG. 6.-The position angles of the minor axes of NGC $636(a)$, NGC $1549(b)$, and NGC $7145(c)$, as a function of radius in arcseconds. The sense of the position angle is the same as normal (increasing east from north), while the zero point is offset by $90^{\circ}$ onto the minor axis. The kinematic angles from the average velocities are indicated by the filled symbols at the error-weighted radius.

Franx and Illingworth 1988; NGC 4406: Illingworth and Franx 1989), it is unlikely that they can be detected by imaging instead of by spectroscopic observations.

The implications of the existence and orientations of these cores for the large-scale figure are not yet clear. It is remarkable that the kinematically misaligned cores are not recognizable as large position angle twists or ellipticity changes in the surface photometry. Although we cannot exclude the possibility that resolution might be a problem for the photometric studies, the flattening and orientation of the isophotes definitely do not change as dramatically as the kinematics, suggesting that the shape of the density (and potential) does not change either. This is supported further by the finding of asymmetric line profiles in the core of IC 1459 by Franx and Illingworth (1988), which suggests that the counterrotating component is cold and contributes relatively little to the light (and density?). Hence the orientation of the angular momentum of the core may be restricted by the shape of the (largescale) potential. In axisymmetric potentials the angular momenta of any orbit can only be parallel or antiparallel to the short axis. More generally, however, orbits with angular momentum aligned with either the shortest or longest axes are naturally allowed in triaxial potentials. Hence the existence of a core which is kinematically misaligned by $90^{\circ}$ from the outer parts of the galaxies may be used as independent evidence that the galaxy NGC 4406 is triaxial.

We may take the last argument even further. We assume that the potential of NGC 4406 has the same orientation at all scales, i.e., the minor axes in the center and in the outer parts are aligned, as are the intermediate and major axes of the center and outer parts. The observation that the rotation in the center is misaligned by $90^{\circ}$ from the rotation in the outer parts suggests that one of the two components is rotating around the intrinsic long axis of the galaxy. In general, kinematic twisting may occur if only one orbit family contributes to the streaming. However, a configuration as seen in NGC 4406 will be very difficult or even impossible to make with only one orbit family contributing to the streaming. Hence, if our assumptions are correct, we have two components with intrinsic rotation axes of which one lies along the major axis and one lies along the minor axis of the figure. We assume first that the rotation in the outer regions is around the intrinsic short axis.
Since we measure a $90^{\circ}$ misalignment between the rotation axis and the apparent minor axis, the line of sight lies in the plane of the intrinsic longest and shortest axis, near to the shortest axis (see, e.g., Binney 1985; FIZ). The rotation axis of the core has to lie also in the plane of the intrinsic longest and shortest axis. Hence it projects to a direction parallel or antiparallel to the rotation of the outer parts, and not to a direction perpendicular to the rotation of the outer parts. This implies that a configuration where the outer parts rotate around the shortest axis is impossible, and we have to conclude that the outer parts of the galaxy rotate around the intrinsic long axis. A very simple and elegant model is one in which the outer parts rotate around the longest axis, and in which the center rotates around the shortest axis. If the line of sight lies near to the intermediate axis then the observed misalignment of $90^{\circ}$ is easily produced.

We have to note that this model relies on assumptions which are not yet certain. If, for example, the core component turns out to be self-gravitating, then the above arguments are not valid. Also, since streamlines within triaxial potentials are complicated, an extensive study of the possible orientations with full dynamical models is necessary. These points have been addressed in Franx (1988) and will be discussed in more detail by FIZ and Franx and de Zeeuw (1989).

It is quite clear that, qualitatively, these data support the now oft-repeated contention that ellipticals are not generically axisymmetric oblate. It is quite reasonable to characterize them as triaxial. The rotation of the center and outer parts of NGC 4406 is easily explained if this galaxy is rotating around its intrinsic long axis in the outer parts. While the fact that some $10 \%$ of ellipticals have $v_{\text {minor }} \gg v_{\text {major }}$ might seem to suggest that all such ellipticals are prolate or prolate-triaxial and rotate around their long axis, it would be unwise to generalize without a careful comparison with projected model galaxies. The same caveat can be applied to any generalization from the fact that the majority of the sample here shows virtually no minor axis rotation. This does not necessarily imply oblateness or near-oblateness. A quantitative assessment is needed and will be provided in a future paper (FIZ).

It is a pleasure to thank Jeanette Barnes and Ed Anderson, who introduced us to IRAF. Discussions with Tim de Zeeuw, 
Roger Davies, and Reynier Peletier are acknowledged. Tim de Zeeuw gave comments on an earlier version of the paper. The Leids Sterrewacht Fonds and the Leiden Kerkhoven-Bosscha
Fonds supported M. F. during part of this work. M. F. wishes to thank Kitt Peak National Observatory and the Space Telescope Science Institute for their hospitality.

\section{APPENDIX A}

\section{FORMAL ERRORS OF THE FOURIER FITTING PROGRAM}

The measured data points of the galaxy are denoted by $G_{i}$. The stellar data points are denoted by $S_{i}$. The stellar data convolved by the broadening function $B$ are denoted by $B S_{i}$. The $\chi^{2}$ value is

$$
\chi^{2}=\sum_{i=1}^{N}\left(G_{i}-B S_{i}\right)^{2} .
$$

The broadening function is parameterized by the values $\left(d_{1}, d_{2}, \ldots, d_{n}\right)$. We linearize $B S_{i}$ with respect to $d_{i}$

$$
B S_{i}=B S_{0, i}+\mathbf{B S}_{1, i}{ }^{T} \mathbf{d}
$$

where the vector $\mathbf{d}$ is given by

$$
\mathbf{d}=\left(\begin{array}{c}
d_{1} \\
d_{2} \\
\vdots \\
d_{n}
\end{array}\right)
$$

and the elements of the vector $\mathbf{B S}_{1, i}$ are

$$
\mathbf{B S}_{1, i}=\frac{\partial}{\partial d_{j}} B S_{i} .
$$

The solution of the linear least-squares solution is standard. We define the matrix $M$

$$
M=\sum_{i=1}^{N} \mathbf{B S}_{1, i} \mathbf{B S}_{1, i}{ }^{T}
$$

The solution for $\boldsymbol{d}$ is given by

$$
\mathrm{d}=M^{-1} \sum_{i=1}^{N}\left(G_{i}-B S_{0, i}\right) \mathrm{BS}_{1, i}
$$

The error matrix is equal to $s^{2} M^{-1}$, with

$$
s^{2}=\frac{1}{N-n} \sum_{i=1}^{N} R_{i}^{2}
$$

and

$$
R_{i}=G_{i}-B S_{0, i}-\mathbf{B S}_{1, i}^{T} \mathbf{d} .
$$

The values are best evaluated in Fourier space, because the convolution is then a multiplication. We define the discrete Fourier transform $\tilde{f}_{j}$ of $f_{i}$ by

$$
\tilde{f}_{j}=\frac{1}{\sqrt{N}} \sum_{i=0}^{N-1} f_{i} e^{-2 \pi i j / N}, \quad j=0,1, \ldots, N-1
$$

and

$$
f_{i}=\frac{1}{\sqrt{N}} \sum_{j=0}^{N-1} \tilde{f}_{j} e^{2 \pi i j / N}, \quad i=0,1, \ldots, N-1 .
$$

We obtain for the linear least-squares fit

$$
\begin{gathered}
M=\sum_{j=0}^{N-1} \tilde{\mathbf{B}}_{1, j}{ }^{*} \tilde{S}_{j}^{*} \tilde{\mathbf{B}}_{1, j}{ }^{T} \tilde{S}_{j}, \\
\mathbf{d}=M^{-1} \sum_{j=0}^{N-1}\left(\tilde{G}_{j}-\tilde{B}_{j} \tilde{S}_{j}\right) * \tilde{\mathbf{B}}_{1, j},
\end{gathered}
$$


and

$$
s^{2}=\frac{1}{N-n} \sum_{j=0}^{N-1} \tilde{R} * \tilde{R} .
$$

The discrete Fourier transform of a real function $f$ has the special property that

$$
\tilde{f}_{N-j}=\tilde{f}_{j}^{*} \text {. }
$$

Hence only the first $N / 2+1$ coefficients are independent. In most application programs, only these coefficients are given. It follows that for the transforms of real functions $f$ and $g$

$$
\sum_{j=0}^{N-1} \tilde{f}_{j}^{*} \tilde{g}_{j}=\tilde{f}_{0} \tilde{g}_{0}+\tilde{f}_{N / 2} \tilde{g}_{N / 2}+\sum_{j=1}^{N / 2-1}\left(\tilde{f}_{j}^{*} \tilde{g}_{j}+\tilde{f}_{j} \tilde{g}_{j}^{*}\right) \text {. }
$$

In practice we fit the stellar spectrum over Fourier frequencies $k$ to $l$, with $0<k<l<N / 2$. The expressions for $M$, d, and $s^{2}$ become

$$
\begin{gathered}
M=\sum_{j=k}^{l} \widetilde{\mathbf{B}}_{1, j}{ }^{*} \widetilde{S}_{j}{ }^{*} \widetilde{\mathbf{B}}_{1, j}{ }^{T} \widetilde{S}_{j}+\widetilde{\mathbf{B}}_{1, j}{\widetilde{S_{j}}}_{\mathbf{B}_{1, j}}{ }^{T}{\widetilde{S_{j}}}^{*}, \\
\mathbf{d}=M^{-1} \sum_{j=k}^{l}\left(\widetilde{G}_{j}-\widetilde{B}_{j} \tilde{S}_{j}\right)^{*} \widetilde{\mathbf{B}}_{1, j}+\left(\widetilde{G}_{j}-\tilde{B}_{j} \widetilde{S}_{j}\right) \tilde{\mathbf{B}}_{1, j}{ }^{*},
\end{gathered}
$$

and

$$
s^{2}=\frac{1}{2(l-k+1)-n} \sum_{j=k}^{l} \widetilde{R}^{*} \tilde{R}+\tilde{R} \widetilde{R}^{*} .
$$

In the old version of the Fourier quotient program, equation (A18) was used to evaluate $s^{2}$, but the right-hand side of equations (A16) and (A17) was divided by a factor of 2 . The solution d remained unchanged, but the error matrix was multiplied by 2 because of this error. Hence the formal errors were a factor of $(2)^{1 / 2}$ too high. We checked the new error estimates with numerical test on "random" spectra. We found excellent agreement between our predicted scatter and the real scatter.

\section{APPENDIX B}

\section{DATA TABLE}

The data are tabulated in Table 7. The rotational velocities and velocity dispersions and their formal errors are given as a function of distance to the center. The radii are given in arcseconds, and negative radii lie toward the west. The radial velocities and velocity dispersions are given in $\mathrm{km} \mathrm{s}^{-1}$. The radii for the rotational velocities and velocity dispersions are different because they are averages weighted by the inverse formal errors of the original data points. The position angles, P.A., are consistent with the normal convention, increasing east from north. 
TABLE 7

ROTATION AND DisPERSION DATA

\begin{tabular}{|c|c|c|c|c|c|c|c|c|c|c|c|}
\hline$r$ & $v_{r}$ & $\Delta v_{r}$ & $r$ & $\sigma$ & $\Delta \sigma$ & $r$ & $v_{r}$ & $\Delta v_{r}$ & $r$ & $\sigma$ & $\Delta \sigma$ \\
\hline \multicolumn{6}{|c|}{ NGC 636 ; P.A. $60^{\circ}$; Major Axis } & \multicolumn{6}{|c|}{ NGC 1395 ; P.A. $93^{\circ}$; Major Axis } \\
\hline 0.8 & 19.4 & 1.8 & 0.7 & 166.3 & 2.3 & -5.2 & -23.8 & 4.4 & -4.7 & 210.6 & 7.8 \\
\hline 2.9 & 51.2 & 4.1 & 2.9 & 171.4 & 4.7 & -7.5 & -25.8 & 7.0 & -6.4 & 211.3 & 7.9 \\
\hline 5.8 & 41.8 & 4.1 & 5.9 & 140.2 & 7.2 & -10.1 & -35.4 & 5.6 & -10.0 & 214.1 & 6.7 \\
\hline 11.5 & 77.1 & 7.2 & 8.9 & 134.3 & 11.9 & -15.5 & -97.8 & 8.1 & -16.6 & 237.7 & 11.1 \\
\hline 22.5 & 48.7 & 12.1 & 19.7 & 163.5 & 12.6 & -22.1 & -92.7 & 9.0 & -27.7 & 169.4 & 10.5 \\
\hline-1.2 & -19.4 & 1.9 & -1.1 & 161.5 & 2.4 & -31.4 & -130.7 & 10.9 & $\ldots$ & $\ldots$ & $\cdots$ \\
\hline-2.8 & -23.5 & 3.6 & -2.8 & 167.0 & 4.3 & \multirow{2}{*}{\multicolumn{6}{|c|}{ NGC 1395 ; P.A. $3^{\circ}$; Minor Axis }} \\
\hline-5.4 & -47.2 & 4.3 & -5.9 & 157.8 & 6.1 & & & & & & \\
\hline-11.3 & -75.4 & 9.1 & -8.2 & 172.2 & 14.0 & & & & & & \\
\hline-23.4 & -81.2 & 12.4 & -21.4 & 132.5 & 16.3 & 0.9 & -2.9 & 2.1 & 1.0 & 247.9 & 2.5 \\
\hline \multirow{2}{*}{\multicolumn{6}{|c|}{ NGC 636 ; P.A. $150^{\circ}$; Minor Axis }} & 2.8 & 9.8 & 3.0 & 2.8 & 235.4 & 4.7 \\
\hline & & & & & & 4.6 & -6.6 & 3.8 & 4.7 & 237.6 & 4.5 \\
\hline 0.9 & -4.6 & 1.1 & 0.9 & 1440 & 15 & 0.9 & -12.0 & 0.1 & 8.1 & 235.2 & 7.1 \\
\hline 2.9 & 6.9 & 2.0 & $\begin{array}{l}0.9 \\
2.8\end{array}$ & 1304 & 1.0 & $\begin{array}{r}9.1 \\
14.2\end{array}$ & 5.1 & 5.8 & 12.4 & 197.3 & 7.4 \\
\hline 5.8 & 5.6 & 2.9 & 5.6 & 109.4 & 0.9 & 14.2 & -12.4 & 5.3 & 24.8 & 221.3 & 9.2 \\
\hline 11.5 & 8.9 & 7.5 & & 130.8 & 4.1 & 18.1 & -28.3 & 18.5 & $\cdots$ & $\cdots$ & $\cdots$ \\
\hline 20.6 & 19.4 & 8.8 & & 108.8 & 13.4 & 31.0 & 5.1 & 8.9 & $\ldots$ & $\ldots$ & $\ldots$ \\
\hline-1.2 & -0.4 & 1.4 & $\ddot{-1}$ & $\cdots$ & $\ldots$ & -1.3 & -2.6 & 2.5 & -1.3 & 261.6 & 2.9 \\
\hline-2.9 & 5.2 & 2.4 & $\begin{array}{r}-1.1 \\
.8\end{array}$ & 153.1 & 2.0 & -3.1 & -8.0 & 2.9 & -2.9 & 252.9 & 3.9 \\
\hline-5.6 & -7.8 & 30 & -2.8 & 155.1 & 3.4 & -5.3 & -1.6 & 3.8 & -4.8 & 249.0 & 5.6 \\
\hline-8.3 & 17.3 & & -5.3 & 144.8 & 4.7 & -7.5 & -27.0 & 5.7 & -6.2 & 227.5 & 6.7 \\
\hline-19.6 & -36.6 & 14.1 & -9.1 & 135.1 & 9.5 & -9.7 & -17.9 & 6.3 & -8.6 & 216.4 & 6.9 \\
\hline & -50.0 & 0.0 & -19.4 & 122.1 & 19.6 & -13.0 & -24.2 & 7.3 & -13.1 & 231.0 & 8.0 \\
\hline \multicolumn{6}{|c|}{ NGC $1379 ;$ P.A. $7^{\circ}$; Major Axis } & $\begin{array}{l}-19.0 \\
-32.2\end{array}$ & $\begin{array}{l}-33.0 \\
-15.6\end{array}$ & $\begin{array}{r}0.8 \\
10.0\end{array}$ & -20.0 & 222.1 & 8.8 \\
\hline 1.1 & 9.2 & 2.6 & 1.0 & 132.2 & 4.7 & \multirow{2}{*}{\multicolumn{6}{|c|}{ NGC 1399 ; P.A. $115^{\circ}$; Major Axis }} \\
\hline 3.0 & 27.0 & 3.9 & 2.8 & 133.6 & 6.2 & & & & & & \\
\hline 5.0 & 26.3 & 3.9 & 4.7 & 104.6 & 8.7 & 1.6 & 16.0 & 6.2 & 1.6 & 341.9 & 7.9 \\
\hline 7.7 & 26.4 & 3.5 & 7.6 & 95.9 & 7.2 & 4.5 & 12.9 & 6.5 & 4.5 & 322.7 & 8.6 \\
\hline 13.8 & 25.6 & 3.6 & 13.7 & 74.2 & 8.7 & 7.7 & 15.4 & 6.1 & 7.6 & 304.6 & 8.3 \\
\hline 27.2 & 15.9 & 7.3 & 30.6 & 78.9 & 14.6 & 13.7 & 24.5 & 4.9 & 14.0 & 252.6 & 8.2 \\
\hline-0.9 & -2.1 & 2.4 & -1.0 & 118.8 & 4.4 & 24.6 & 25.5 & 9.2 & 24.7 & 233.2 & 15.7 \\
\hline-2.9 & -12.8 & 3.1 & -2.8 & 112.6 & 7.7 & 35.1 & 44.8 & 21.3 & 37.3 & 315.3 & 34.2 \\
\hline-4.9 & -25.1 & 3.9 & -4.8 & 100.9 & 7.4 & -1.6 & 5.0 & 7.0 & -1.5 & 341.8 & 9.1 \\
\hline-7.4 & -14.0 & 3.8 & -8.4 & 98.5 & 8.0 & -4.4 & 4.9 & 5.9 & -4.4 & 290.1 & 8.3 \\
\hline-13.4 & -24.8 & 3.5 & -15.6 & 75.1 & 8.6 & -8.1 & -23.0 & 6.0 & -7.6 & 274.0 & 9.5 \\
\hline-25.8 & -14.8 & 6.5 & -34.6 & 78.6 & 18.0 & -14.2 & -24.2 & 5.1 & -13.8 & 252.5 & 8.0 \\
\hline \multirow{2}{*}{\multicolumn{6}{|c|}{ NGC $1379 ;$ P.A. $97^{\circ} ;$ Minor Axis }} & -24.4 & -29.1 & 8.9 & -25.2 & 174.4 & 15.4 \\
\hline & & & & & & -35.3 & -26.2 & 14.7 & -35.3 & 244.9 & 30.7 \\
\hline 0.8 & 7.2 & 2.6 & 0.8 & 136.3 & 4.2 & \multicolumn{6}{|c|}{ NGC 1399 ; P.A. $25^{\circ}$; Minor Axis } \\
\hline 2.8 & 4.1 & 3.1 & 2.7 & 124.8 & 5.8 & & & & & & \\
\hline 5.0 & -4.7 & 3.8 & 4.6 & 121.4 & 9.1 & 1.5 & 33.5 & 7.5 & 1.6 & 353.6 & 9.2 \\
\hline 7.9 & -10.1 & 3.9 & 7.3 & 106.2 & 6.7 & 4.4 & 31.3 & 5.7 & 4.4 & 317.7 & 7.5 \\
\hline 13.8 & -5.1 & 3.5 & 14.6 & 91.3 & 7.6 & 8.0 & -7.0 & 5.6 & 8.0 & 284.3 & 8.0 \\
\hline 24.3 & -5.4 & 8.7 & 34.3 & 116.4 & 17.7 & 14.0 & -5.5 & 5.3 & 14.2 & 246.8 & 8.9 \\
\hline 35.7 & 4.4 & 14.1 & $\ldots$ & $\ldots$ & $\ldots$ & 24.1 & -3.2 & 9.2 & 23.3 & 225.9 & 18.5 \\
\hline-1.1 & -4.2 & 2.7 & -1.1 & 132.6 & 4.4 & 35.4 & -18.3 & 14.8 & 34.5 & 220.8 & 20.8 \\
\hline-3.1 & -5.1 & 3.1 & -3.0 & 117.5 & 6.1 & -1.7 & 30.0 & 6.1 & -1.7 & 329.3 & 7.9 \\
\hline-5.2 & -8.9 & 4.3 & -4.9 & 119.7 & 9.5 & -4.7 & 34.9 & 5.9 & -4.7 & 282.1 & 8.4 \\
\hline-7.9 & 4.7 & 3.3 & -7.9 & 99.1 & 6.8 & -7.8 & 23.3 & 5.1 & -7.8 & 237.6 & 8.3 \\
\hline-14.5 & 9.1 & 3.6 & -13.5 & 104.8 & 10.3 & -14.0 & -0.3 & 5.1 & -13.9 & 241.4 & 8.5 \\
\hline-24.4 & -10.0 & 7.5 & -21.8 & 50.4 & 11.9 & -24.4 & 18.7 & 9.4 & -25.2 & 250.5 & 14.9 \\
\hline-34.6 & 0.5 & 10.3 & $\ldots$ & $\ldots$ & $\ldots$ & -36.0 & 14.0 & 16.5 & -38.5 & 289.9 & 27.2 \\
\hline \multicolumn{6}{|c|}{ NGC 1395 ; P.A. $93^{\circ}$; Major Axis } & \multicolumn{6}{|c|}{ NGC 1404; P.A. $164^{\circ}$; Major Axis } \\
\hline 0.8 & 8.8 & 2.6 & 0.8 & 248.4 & 3.1 & 1.5 & 18.7 & 3.5 & 1.5 & 245.4 & 5.4 \\
\hline 3.1 & 47.5 & 4.1 & 3.0 & 265.4 & 5.6 & 4.6 & 33.4 & 4.7 & 4.6 & 265.3 & 7.2 \\
\hline 5.2 & 49.6 & 5.5 & 4.6 & 252.2 & 8.1 & 7.8 & 57.3 & 4.5 & 7.5 & 259.2 & 8.0 \\
\hline 7.1 & 34.3 & 7.2 & 6.3 & 219.0 & 8.2 & 13.4 & 88.0 & 4.6 & 13.2 & 227.1 & 7.4 \\
\hline 9.7 & 60.0 & 5.8 & 9.9 & 208.6 & 6.8 & 24.2 & 89.3 & 7.9 & 22.9 & 236.6 & 15.2 \\
\hline 15.3 & 89.2 & 6.5 & 16.6 & 240.7 & 9.1 & 38.3 & 97.1 & 13.1 & 38.9 & 150.3 & 20.2 \\
\hline 22.5 & 125.9 & 8.8 & 28.0 & 220.4 & 10.8 & -1.6 & 15.8 & 3.4 & -1.6 & 227.9 & 5.6 \\
\hline 32.0 & 138.5 & 16.7 & $\ldots$ & $\ldots$ & $\ldots$ & -4.5 & -32.1 & 4.0 & -4.4 & 255.1 & 6.1 \\
\hline-1.2 & -11.0 & 3.0 & -1.2 & 246.6 & 3.6 & -8.1 & -71.8 & 4.9 & -8.3 & 249.1 & 8.0 \\
\hline-3.0 & -29.9 & 3.5 & -2.9 & 240.1 & 4.8 & -14.0 & -82.3 & 4.3 & -14.1 & 223.9 & 8.0 \\
\hline
\end{tabular}


TABLE 7-Continued

\begin{tabular}{|c|c|c|c|c|c|c|c|c|c|c|c|}
\hline$r$ & $v_{r}$ & $\Delta v_{r}$ & $r$ & $\sigma$ & $\Delta \sigma$ & $r$ & $v_{r}$ & $\Delta v_{r}$ & $r$ & $\sigma$ & $\Delta \sigma$ \\
\hline \multicolumn{6}{|c|}{ NGC 1404; P.A. $164^{\circ}$; Major Axis } & \multicolumn{6}{|c|}{ NGC 1439; P.A. $128^{\circ}$; Minor Axis } \\
\hline-25.5 & -115.8 & 8.7 & -22.7 & 220.7 & 15.5 & 12.7 & 0.3 & 6.1 & 15.4 & 77.9 & 14.0 \\
\hline-37.8 & -109.4 & 13.8 & -39.4 & 138.4 & 20.0 & 21.4 & 5.7 & 11.7 & 21.3 & 129.8 & 25.3 \\
\hline \multirow{2}{*}{\multicolumn{6}{|c|}{ NGC 1404; P.A. $74^{\circ}$; Minor Axis }} & -0.5 & -1.2 & 3.2 & -0.5 & 152.4 & 4.7 \\
\hline & & & & & & -1.4 & 5.8 & 4.1 & -1.5 & 156.3 & 5.6 \\
\hline 1.4 & 23.4 & 2.9 & 1.4 & 236.4 & 4.7 & $\begin{array}{l}-2.8 \\
-4.7\end{array}$ & -1.3 & 3.7 & $\begin{array}{l}-2.7 \\
-4.6\end{array}$ & $\begin{array}{l}139.6 \\
131.3\end{array}$ & $\begin{array}{l}7.2 \\
7.9\end{array}$ \\
\hline 4.4 & -1.1 & 3.9 & 4.4 & 256.2 & 6.1 & -7.7 & $\begin{array}{r}1.0 \\
-0.9\end{array}$ & 5.4 & $\begin{array}{l}-4.6 \\
-8.8\end{array}$ & $\begin{array}{l}131.3 \\
117.6\end{array}$ & $\begin{array}{r}7.9 \\
10.4\end{array}$ \\
\hline 7.8 & 10.5 & 3.7 & 7.8 & 223.1 & 6.6 & -14.1 & $\begin{array}{l}-0.9 \\
-2.5\end{array}$ & $\begin{array}{l}5.0 \\
5.6\end{array}$ & $\begin{array}{r}-8.8 \\
-15.4\end{array}$ & 61.2 & $\begin{array}{l}10.4 \\
17.3\end{array}$ \\
\hline 15.1 & 9.8 & 4.3 & 15.2 & 233.6 & 7.5 & -21.7 & -20.2 & 10.9 & $\ldots$ & & \\
\hline 31.9 & -6.6 & 12.1 & 31.4 & 226.3 & 20.7 & & & & & & \\
\hline-1.5 & 18.7 & 3.0 & -1.4 & 256.9 & 4.7 & \multicolumn{6}{|c|}{ NGC 1549; P.A. $127^{\circ}$; Major Axis } \\
\hline-4.4 & -2.0 & 3.8 & -4.4 & 256.4 & 5.8 & & & & & & \\
\hline-7.6 & -3.9 & 4.2 & -7.5 & 249.3 & 6.5 & 1.4 & 46.7 & 3.1 & 1.4 & 208.9 & 5.5 \\
\hline-15.4 & -10.6 & 4.0 & -16.3 & 216.4 & 7.3 & 4.3 & 27.0 & 3.3 & 4.0 & 224.3 & 6.1 \\
\hline-31.4 & 15.2 & 10.6 & -31.3 & 203.5 & 24.6 & 7.9 & 20.5 & 4.0 & 7.3 & 196.5 & 6.8 \\
\hline \multirow{2}{*}{\multicolumn{6}{|c|}{ NGC 1407; P.A. $58^{\circ}$; Major Axis }} & 14.2 & 25.4 & 4.0 & 13.3 & 165.6 & 7.3 \\
\hline & & & & & & 24.6 & 47.7 & 7.4 & 22.9 & 210.6 & 12.2 \\
\hline & & & & & & 38.3 & 78.6 & 9.1 & 37.6 & 190.8 & 16.3 \\
\hline 1.3 & 18.5 & 4.9 & 1.3 & 290.0 & 6.8 & -1.4 & 8.8 & 3.2 & -1.4 & 205.0 & 5.8 \\
\hline 4.3 & -14.5 & 5.9 & 4.2 & 282.4 & 9.1 & -4.3 & -4.6 & 3.1 & -4.3 & 211.7 & 5.5 \\
\hline 7.7 & -10.9 & 6.9 & 7.9 & 260.2 & 10.6 & -7.9 & -26.6 & 4.1 & -8.3 & 202.5 & 7.6 \\
\hline 13.2 & -27.0 & 8.8 & 12.8 & 247.4 & 16.2 & -14.2 & -31.2 & 4.1 & -14.9 & 174.9 & 7.7 \\
\hline 19.4 & -49.1 & 12.0 & 19.1 & 231.8 & 17.2 & -24.5 & -70.9 & 6.3 & -23.9 & 144.6 & 12.6 \\
\hline 28.4 & -77.3 & 17.1 & 33.7 & 256.3 & 41.2 & -37.2 & -82.7 & 8.8 & -35.5 & 181.4 & 14.5 \\
\hline-1.4 & 5.5 & 5.1 & -1.4 & 280.9 & 7.4 & -50.8 & -57.9 & 24.4 & -50.1 & 117.9 & 29.2 \\
\hline-4.2 & 10.7 & 5.6 & -4.3 & 269.1 & 8.4 & & & & & & \\
\hline-7.5 & 16.0 & 7.2 & -8.3 & 272.0 & 11.4 & \multicolumn{6}{|c|}{ NGC 1549 ; P.A. $37^{\circ}$; Minor Axis } \\
\hline-12.5 & $\begin{array}{l}29.9 \\
262\end{array}$ & 8.9 & -13.0 & 261.9 & 16.3 & & & & & & \\
\hline-19.6 & 26.2 & 9.7 & -19.5 & 217.3 & 16.6 & 1.4 & 48.2 & 2.6 & 1.5 & 219.4 & 3.9 \\
\hline-29.6 & 75.5 & 17.1 & -34.3 & 203.4 & 37.3 & 4.2 & 23.5 & 3.3 & 4.2 & 223.9 & 5.3 \\
\hline & & & & & & 7.9 & 29.0 & 3.7 & 7.9 & 200.4 & 6.7 \\
\hline & NGC & 107; P.A & $8^{\circ} ;$ Mino & Axis & & 14.0 & 33.2 & 4.1 & 13.9 & 178.1 & 7.1 \\
\hline & & & & & & 24.2 & 57.2 & 6.1 & 24.7 & 180.5 & 10.1 \\
\hline 1.3 & -4.9 & 5.0 & 1.3 & 303.5 & 6.9 & 36.9 & 49.6 & 9.2 & 38.7 & 147.2 & 14.8 \\
\hline 4.2 & -24.8 & 5.1 & 4.1 & 288.4 & 7.4 & -1.6 & 26.2 & 3.0 & -1.5 & 208.1 & 4.5 \\
\hline 7.7 & -2.3 & 6.1 & 7.6 & 273.1 & 10.1 & -4.3 & 1.4 & 3.1 & -4.3 & 209.1 & 5.1 \\
\hline 13.7 & -18.8 & 7.7 & 14.7 & 268.9 & 11.0 & -7.7 & -10.9 & 3.6 & -7.6 & 186.6 & 6.5 \\
\hline 23.9 & -35.3 & 11.1 & 29.7 & 190.9 & 23.1 & -13.8 & -34.3 & 4.0 & -13.5 & 182.9 & 6.9 \\
\hline 33.0 & -7.8 & 16.8 & 34.1 & 232.1 & 34.4 & -24.6 & -51.2 & 5.7 & -24.6 & 178.8 & 8.7 \\
\hline-1.3 & -11.4 & 4.4 & -1.3 & 264.7 & 6.5 & -38.6 & -70.7 & 8.5 & -40.6 & 133.1 & 14.3 \\
\hline-4.2 & 7.4 & 4.8 & -4.1 & 259.1 & 7.6 & \multirow{2}{*}{\multicolumn{6}{|c|}{ NGC 1700; P.A. $91^{\circ}$; Major Axis }} \\
\hline-7.7 & 10.9 & 6.8 & -7.6 & 264.2 & 11.1 & & & & & & \\
\hline-13.4 & 16.3 & 7.0 & -14.2 & 254.8 & 10.1 & & & & & & \\
\hline-24.8 & 10.1 & 11.5 & -29.8 & 235.4 & 31.1 & 0.8 & 3.8 & 1.9 & 0.8 & 228.2 & 2.4 \\
\hline-33.4 & 5.4 & 21.1 & -33.5 & 263.0 & 29.1 & 2.8 & 19.4 & 4.1 & 2.9 & 230.8 & 5.9 \\
\hline & & & & & & 4.5 & -22.5 & 5.5 & 5.0 & 237.5 & 7.7 \\
\hline & NGC & 439; P. & ${ }^{\circ}$; Major & Axis & & 6.9 & -68.4 & 7.8 & 6.4 & 230.8 & 14.3 \\
\hline & & & & & & 11.9 & -80.5 & 10.0 & 9.2 & 256.4 & 12.4 \\
\hline 0.5 & -2.6 & 4.4 & 0.5 & 160.4 & 6.2 & 20.0 & -93.8 & 10.0 & 19.3 & 192.3 & 11.8 \\
\hline 1.4 & -24.6 & 4.0 & 1.4 & 149.0 & 6.0 & -1.2 & -19.2 & 2.4 & -1.1 & 243.9 & 3.1 \\
\hline 2.9 & -24.5 & 4.0 & 2.7 & 152.9 & 6.4 & -2.8 & -15.5 & 3.9 & -2.8 & 244.5 & 3.9 \\
\hline 4.8 & -23.1 & 6.0 & 4.8 & 134.2 & 8.6 & -4.5 & 27.3 & 5.4 & -5.0 & 265.0 & 11.1 \\
\hline 7.2 & -32.3 & 5.5 & 8.3 & 106.6 & 11.8 & -6.8 & 71.2 & 7.6 & -7.1 & 261.5 & 8.2 \\
\hline 13.1 & -7.7 & 9.1 & 14.7 & 158.3 & 19.1 & -11.6 & 63.3 & 7.7 & -11.5 & 218.6 & 12.5 \\
\hline 24.6 & 17.5 & 10.4 & 26.0 & 72.6 & 23.0 & -21.5 & 93.6 & 12.1 & $\ldots$ & $\ldots$ & $\ldots$ \\
\hline-0.4 & 11.5 & 4.6 & -0.4 & 148.4 & 7.0 & & & & & & \\
\hline-1.4 & 22.6 & 3.8 & -1.4 & 155.4 & 5.6 & & NG & $1700 ; \mathrm{P}$ & ${ }^{\circ}$; Minor & Axis & \\
\hline-3.0 & 23.7 & 3.5 & -3.0 & 133.8 & 6.3 & & & & & & 2.2 \\
\hline $\begin{array}{l}-4.8 \\
-7.2\end{array}$ & $\begin{array}{r}19.8 \\
8.7\end{array}$ & $\begin{array}{l}6.3 \\
6.3\end{array}$ & $\begin{array}{l}-5.0 \\
-8.2\end{array}$ & $\begin{array}{l}137.9 \\
132.4\end{array}$ & $\begin{array}{r}9.7 \\
11.9\end{array}$ & $\begin{array}{l}0.8 \\
2.9\end{array}$ & $\begin{array}{l}-2.1 \\
14.5\end{array}$ & $\begin{array}{l}1.8 \\
3.4\end{array}$ & $\begin{array}{l}0.8 \\
2.8\end{array}$ & $\begin{array}{l}24.3 \\
239.9\end{array}$ & $\begin{array}{l}2.2 \\
5.2\end{array}$ \\
\hline-12.6 & -11.7 & 6.7 & -18.1 & 117.8 & 12.1 & 4.9 & 13.0 & 6.1 & 4.3 & 217.5 & 6.8 \\
\hline-22.8 & -18.2 & 10.4 & 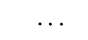 & & .. & 7.2 & 1.3 & 6.8 & 7.2 & 217.3 & 8.1 \\
\hline & & & & & & 12.6 & -10.8 & 6.9 & 18.6 & 188.2 & 11.0 \\
\hline & NGC & 39; P.A & Mino & Axis & & 17.9 & 16.8 & 18.3 & & & \\
\hline & & & & & & -1.3 & 5.0 & 2.1 & -1.2 & 235.0 & 2.7 \\
\hline 0.4 & . & 0.0 & 0.5 & 147.4 & 5.2 & -2.9 & -1.6 & 3.6 & -2.8 & 257.7 & 4.3 \\
\hline 1.4 & -10.0 & 3.4 & 1.4 & 169.8 & 5.0 & -4.6 & 21.5 & 5.8 & -4.3 & 252.9 & 7.8 \\
\hline 3.0 & -3.4 & 4.0 & 2.7 & 156.1 & 6.2 & -7.0 & -9.0 & 7.4 & -7.0 & 229.6 & 9.3 \\
\hline 5.0 & -8.1 & 5.4 & 4.6 & 135.7 & 7.8 & -10.9 & 3.9 & 7.4 & -9.7 & 162.3 & 12.5 \\
\hline 7.9 & -0.9 & 6.0 & 8.0 & 127.0 & 11.3 & -22.1 & -5.5 & 11.4 & -19.6 & 196.2 & 12.0 \\
\hline
\end{tabular}


TABLE 7.-Continued

\begin{tabular}{|c|c|c|c|c|c|c|c|c|c|c|c|}
\hline$r$ & $v_{r}$ & $\Delta v_{r}$ & $r$ & $\sigma$ & $\Delta \sigma$ & $r$ & $v_{r}$ & $\Delta v_{r}$ & $r$ & $\sigma$ & $\Delta \sigma$ \\
\hline \multicolumn{6}{|c|}{ NGC 3091 ; P.A. $147^{\circ}$; Major Axis } & \multicolumn{6}{|c|}{ NGC 3557 ; P.A. $129^{\circ}$; Minor Axis } \\
\hline 0.6 & 16.0 & 17.1 & 0.5 & 354.5 & 15.1 & 0.7 & -2.7 & 12.4 & 0.7 & 231.1 & 14.6 \\
\hline 1.2 & -12.3 & 9.1 & 1.2 & 322.3 & 7.7 & 1.3 & -7.6 & 5.1 & 1.3 & 262.4 & 5.3 \\
\hline 3.2 & -48.3 & 11.1 & 3.1 & 296.6 & 11.8 & 3.3 & 6.5 & 6.7 & 3.2 & 245.4 & 7.5 \\
\hline 6.1 & -78.0 & 13.4 & 6.5 & 252.7 & 14.3 & 6.2 & -17.5 & 9.3 & 5.8 & 246.4 & 11.4 \\
\hline 14.5 & -96.0 & 29.6 & $\ldots$ & $\ldots$ & $\ldots$ & 16.5 & -4.3 & 19.6 & 12.2 & 215.4 & 21.3 \\
\hline-0.8 & 13.0 & 7.9 & -0.8 & 325.4 & 6.6 & -0.7 & -2.5 & 4.5 & -0.7 & 256.1 & 4.7 \\
\hline-1.4 & 25.4 & 22.9 & -1.4 & 320.3 & 21.6 & -1.2 & -10.5 & 16.1 & -1.2 & 244.8 & 18.3 \\
\hline-3.3 & 32.9 & 9.0 & -3.4 & 285.0 & 9.3 & -3.2 & -4.3 & 5.5 & -2.7 & 255.9 & 6.8 \\
\hline-7.8 & 45.9 & 19.2 & -8.4 & 258.0 & 20.4 & -7.3 & -5.2 & 12.8 & -5.4 & 238.3 & 10.0 \\
\hline-14.2 & 96.3 & 30.3 & $\ldots$ & $\ldots$ & $\ldots$ & -15.1 & -37.5 & 16.4 & -12.5 & 225.6 & 18.9 \\
\hline \multicolumn{6}{|c|}{ NGC 3091; P.A. $57^{\circ}$; Minor Axis } & \multicolumn{6}{|c|}{ NGC 4406 ; P.A. $120^{\circ}$; Major Axis } \\
\hline 0.4 & 0.8 & 8.4 & 0.4 & 334.1 & 6.7 & 0.5 & 34.7 & 9.8 & 0.5 & 230.7 & 11.6 \\
\hline 2.8 & 7.0 & 9.2 & 2.8 & 299.8 & 9.0 & 1.9 & 46.7 & 3.6 & 1.9 & 230.7 & 4.1 \\
\hline 9.5 & 45.8 & 13.9 & 8.5 & 191.5 & 20.6 & 3.8 & 19.7 & 4.5 & 3.9 & 218.1 & 5.7 \\
\hline-1.5 & 11.2 & 7.9 & -1.5 & 301.1 & 7.2 & 7.5 & 4.5 & 4.6 & 7.5 & 220.5 & 5.7 \\
\hline-4.1 & -2.9 & 13.2 & -4.1 & 285.4 & 14.4 & 17.4 & 2.8 & 5.6 & 16.1 & 199.7 & 7.6 \\
\hline-10.8 & 40.3 & 24.4 & -8.5 & 214.2 & 38.1 & -0.1 & -12.4 & 3.6 & -0.1 & 249.4 & 3.6 \\
\hline \multirow{2}{*}{\multicolumn{6}{|c|}{ NGC 3379; P.A. $68^{\circ}$; Major Axis }} & -1.5 & -75.2 & 13.9 & -1.7 & 238.8 & 13.7 \\
\hline & & & & & & -2.8 & -41.5 & 3.1 & -2.8 & 234.5 & 3.5 \\
\hline 0.9 & & 1.6 & 0.9 & & & $\begin{array}{r}-6.9 \\
-154\end{array}$ & -0.9 & 5.2 & $\begin{array}{r}-6.9 \\
-14.8\end{array}$ & 223.6 & 6.3 \\
\hline 3.0 & -19.5 & 1.9 & 3.0 & $\begin{array}{l}228.3 \\
195.4\end{array}$ & $\begin{array}{l}1.6 \\
2.4\end{array}$ & -15.4 & 4.3 & 5.5 & -14.8 & 207.1 & 7.0 \\
\hline 4.9 & -27.9 & 2.1 & 5.1 & 181.4 & 3.0 & \multirow{2}{*}{\multicolumn{6}{|c|}{ NGC 4406; P.A. $30^{\circ}$; Minor Axis }} \\
\hline 7.1 & -47.3 & 3.9 & 7.5 & 186.0 & 5.9 & & & & & & \\
\hline 9.3 & -31.7 & 3.5 & 9.5 & 180.3 & 5.3 & 1.6 & -11.2 & 3.4 & 1.6 & 234.5 & 3.8 \\
\hline 13.6 & -52.6 & 3.3 & 14.2 & 155.4 & 5.7 & 3.6 & -3.4 & 4.2 & 3.6 & 221.7 & 5.1 \\
\hline 22.4 & -42.5 & 5.0 & 18.4 & 150.9 & 8.0 & 7.1 & -9.8 & 4.2 & 7.1 & 205.6 & 5.3 \\
\hline 32.3 & -65.4 & 6.1 & 31.4 & 130.1 & 8.8 & 14.3 & -20.5 & 7.9 & 14.1 & 219.8 & 9.6 \\
\hline-1.2 & 15.1 & 1.7 & -1.2 & 213.3 & 1.9 & 26.6 & -29.5 & 15.3 & 25.9 & 247.0 & 20.8 \\
\hline-3.0 & 36.8 & 2.0 & -3.1 & 204.0 & 2.4 & -0.4 & -9.4 & 3.4 & -0.4 & 242.5 & 3.6 \\
\hline-5.0 & 41.9 & 2.1 & -5.1 & 195.4 & 2.8 & -3.1 & 9.3 & 3.0 & -3.1 & 218.5 & 3.7 \\
\hline-7.1 & 53.5 & 3.8 & -7.5 & 188.2 & 5.7 & -7.1 & 11.1 & 5.5 & -7.1 & 217.1 & 6.7 \\
\hline-9.3 & 33.0 & 2.8 & -9.3 & 145.8 & 5.0 & -12.4 & 32.7 & 7.3 & -12.9 & 211.3 & 8.8 \\
\hline-13.6 & 43.0 & 3.1 & -13.9 & 158.7 & 5.3 & -24.6 & 70.4 & 15.1 & -27.3 & 243.9 & 25.8 \\
\hline-22.4 & 51.4 & 5.9 & -18.6 & 172.1 & 7.7 & & & & & & \\
\hline-33.0 & 64.9 & 8.6 & -32.9 & 184.1 & 10.2 & \multicolumn{6}{|c|}{ NGC 4472 ; P.A. $162^{\circ}$; Major Axis } \\
\hline \multicolumn{6}{|c|}{ NGC 3379 ; P.A. $158^{\circ}$; Minor Axis } & 0.5 & -9.2 & 9.6 & 0.5 & 292.9 & 9.7 \\
\hline & & & & & & 1.5 & -8.8 & 10.9 & 1.5 & 295.4 & 10.9 \\
\hline 0.9 & -12.6 & 1.5 & 0.9 & 228.1 & 1.5 & 3.2 & -14.2 & 8.7 & 3.3 & 293.1 & 8.6 \\
\hline 3.1 & 8.8 & 1.8 & 3.1 & 207.5 & 2.0 & 6.7 & 8.1 & 13.4 & 7.0 & 291.5 & 14.3 \\
\hline 5.1 & 6.3 & 2.2 & 5.2 & 194.7 & 3.0 & 14.5 & 38.7 & 15.1 & 14.9 & 275.4 & 25.2 \\
\hline 7.2 & -13.3 & 3.7 & 7.4 & 165.9 & 5.0 & -0.5 & -8.7 & 8.7 & -0.5 & 291.0 & 8.9 \\
\hline 9.8 & -8.5 & 3.3 & 9.8 & 188.4 & 5.3 & -1.5 & 9.9 & 9.8 & -1.5 & 285.6 & 10.0 \\
\hline 14.1 & -11.1 & 5.6 & 13.8 & 189.8 & 6.0 & -3.2 & 3.1 & 8.6 & -3.3 & 298.2 & 8.4 \\
\hline 20.2 & -1.3 & 5.1 & 24.0 & 135.5 & 7.2 & -6.8 & -36.5 & 13.2 & -6.7 & 296.4 & 14.4 \\
\hline 31.9 & -23.6 & 6.7 & $\ldots$ & $\ldots$ & $\ldots$ & -15.0 & -34.9 & 13.1 & -16.0 & 232.8 & 20.8 \\
\hline-1.2 & -9.3 & 1.6 & -1.2 & 211.1 & 1.8 & \multirow{2}{*}{\multicolumn{6}{|c|}{ NGC 4472 ; P.A. $72^{\circ}$; Minor Axis }} \\
\hline-3.1 & -4.7 & 1.8 & -3.0 & 201.0 & 2.3 & & & & & & \\
\hline-5.0 & 1.0 & 2.3 & -4.8 & 196.7 & 3.0 & & & & & & \\
\hline-7.2 & 8.9 & 3.5 & -7.1 & 176.5 & 4.5 & 0.5 & 4.8 & 10.5 & 0.5 & 295.4 & $\begin{array}{l}10.4 \\
12.6\end{array}$ \\
\hline-9.8 & 11.2 & 3.0 & -9.4 & 164.5 & 5.3 & 1.5 & 8.0 & 12.6 & 1.4 & 294.9 & $\begin{array}{r}12.6 \\
9.5\end{array}$ \\
\hline-14.4 & -0.4 & 5.4 & -13.3 & 184.0 & 5.9 & 3.2 & 10.7 & 9.6 & 3.4 & 275.6 & 9.5 \\
\hline-21.0 & -33.9 & 6.2 & -22.6 & 169.0 & 7.2 & 6.8 & -4.8 & 13.9 & 7.6 & 295.8 & 15.5 \\
\hline-33.4 & -4.7 & 7.4 & $\cdots$ & $\cdots$ & 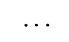 & 15.1 & 23.7 & 19.7 & 13.0 & 240.1 & 27.0 \\
\hline & & & & & & -0.5 & -6.2 & 10.4 & -0.5 & 296.6 & 10.3 \\
\hline & NGC & $557 ; \mathrm{P}$ & ; Majo & Axis & & -1.5 & -11.7 & 11.7 & -1.5 & 285.1 & 12.0 \\
\hline & & & & & & -3.3 & 1.0 & 10.9 & -3.5 & 294.2 & 10.4 \\
\hline 0.5 & -50.0 & 5.1 & 0.5 & 251.5 & 5.6 & -6.8 & 17.4 & 15.2 & -7.3 & 287.4 & 18.4 \\
\hline 1.5 & -107.0 & 6.0 & 1.5 & 257.3 & 6.5 & -14.2 & 1.3 & 19.1 & -12.1 & 261.5 & 27.0 \\
\hline 3.1 & -134.8 & 5.3 & 2.8 & 243.7 & 6.7 & & & & & & \\
\hline 6.7 & -142.6 & 9.9 & 5.5 & 222.6 & 9.2 & & NG & $649 ; \mathrm{P}$ & $2^{\circ} ; \mathrm{Ma}$ & Axis & \\
\hline 14.8 & -135.2 & 14.8 & 14.5 & 209.7 & 20.4 & & & & & & \\
\hline-0.5 & 56.0 & 5.2 & -0.5 & 257.3 & 5.7 & 0.5 & -32.7 & 14.1 & 0.5 & 352.6 & 12.4 \\
\hline-1.4 & 110.7 & 5.5 & -1.4 & 248.8 & 6.2 & 1.5 & -38.8 & 14.1 & 1.5 & 358.7 & 12.2 \\
\hline-3.1 & 141.1 & 6.2 & -2.8 & 265.9 & 7.3 & 3.3 & -44.1 & 10.1 & 3.3 & 335.3 & 9.1 \\
\hline-6.7 & 146.4 & 9.8 & -5.3 & 240.9 & 9.6 & 6.9 & -49.5 & 9.8 & 7.1 & 274.5 & 10.5 \\
\hline-15.9 & 166.3 & 17.9 & -13.9 & 254.9 & 20.2 & 15.1 & -62.2 & 12.9 & 15.4 & 255.7 & 17.1 \\
\hline
\end{tabular}


TABLE 7-Continued

\begin{tabular}{|c|c|c|c|c|c|c|c|c|c|c|c|}
\hline$r$ & $v_{r}$ & $\Delta v_{r}$ & $r$ & $\sigma$ & $\Delta \sigma$ & $r$ & $v_{r}$ & $\Delta v_{r}$ & $r$ & $\sigma$ & $\Delta \sigma$ \\
\hline \multicolumn{6}{|c|}{ NGC 4649 ; P.A. $102^{\circ}$; Major Axis } & \multicolumn{6}{|c|}{ NGC $7144 ;$ P.A. $27^{\circ}$; Major Axis } \\
\hline-0.5 & 17.5 & 12.3 & -0.5 & 341.9 & 11.5 & 12.9 & 34.1 & 6.2 & 12.4 & 149.4 & 11.8 \\
\hline-1.5 & 9.9 & 13.4 & -1.5 & 344.1 & 12.8 & 23.5 & 29.2 & 9.9 & 28.3 & 143.7 & 18.3 \\
\hline-3.3 & 40.1 & 8.8 & -3.2 & 317.0 & 8.1 & -1.2 & -0.8 & 2.5 & -1.0 & 178.4 & 4.5 \\
\hline-6.9 & 39.1 & 11.3 & -6.7 & 305.5 & 12.2 & -4.3 & -32.7 & 4.4 & -4.0 & 166.0 & 7.9 \\
\hline \multirow[t]{2}{*}{-15.3} & 43.1 & 11.2 & -14.1 & 238.2 & 13.7 & -7.5 & -30.0 & 6.1 & -7.0 & 137.5 & 10.9 \\
\hline & \multicolumn{5}{|c|}{ NGC 4649; P.A. $12^{\circ}$; Minor Axis } & $\begin{array}{l}-14.1 \\
-28.0\end{array}$ & $\begin{array}{l}-50.5 \\
-36.0\end{array}$ & $\begin{array}{r}5.5 \\
12.0\end{array}$ & $\begin{array}{l}-12.1 \\
-28.5\end{array}$ & $\begin{array}{l}149.1 \\
166.2\end{array}$ & $\begin{array}{l}11.4 \\
18.7\end{array}$ \\
\hline 0.5 & 12.9 & 8.7 & 0.5 & 366.9 & 5.9 & \multicolumn{6}{|c|}{ NGC 7144 ; P.A. $117^{\circ}$; Minor Axis } \\
\hline 1.6 & 11.2 & 16.5 & 1.6 & 360.3 & 14.1 & & & & & & \\
\hline 3.4 & 10.4 & 5.7 & 3.3 & 325.8 & 4.7 & 1.3 & 2.1 & 2.2 & 1.4 & 171.8 & 3.5 \\
\hline 7.2 & -5.0 & 6.7 & 7.3 & 278.4 & 6.7 & 4.3 & 6.9 & 3.7 & 4.3 & 170.9 & 6.7 \\
\hline 15.0 & 12.9 & 7.8 & 14.4 & 263.6 & 9.1 & 7.7 & -3.2 & 4.6 & 8.0 & 130.4 & 7.7 \\
\hline-0.4 & -3.2 & 14.6 & -0.4 & 350.6 & 12.8 & 13.6 & -11.1 & 5.9 & 14.0 & 136.7 & 12.2 \\
\hline-1.5 & 11.0 & 8.3 & -1.5 & 348.6 & 6.2 & 22.4 & -22.3 & 14.2 & 25.2 & 165.9 & 26.1 \\
\hline-3.4 & 6.8 & 7.2 & -3.4 & 335.4 & 5.7 & -1.3 & 1.5 & 2.2 & -1.3 & 167.0 & 3.6 \\
\hline-7.0 & 4.7 & 6.1 & -7.0 & 291.7 & 5.9 & -4.3 & -2.9 & 3.4 & -4.5 & 153.7 & 6.4 \\
\hline \multirow[t]{3}{*}{-15.5} & -18.4 & 8.2 & -14.9 & 256.0 & 9.9 & -7.7 & -0.3 & 4.9 & -8.1 & 153.0 & 9.6 \\
\hline & \multirow{2}{*}{\multicolumn{5}{|c|}{ IC 4296; P.A. $60^{\circ}$; Major Axis }} & -13.2 & 7.7 & 5.9 & -14.5 & 135.9 & 11.4 \\
\hline & & & & & & -21.8 & 4.6 & 11.7 & -23.3 & 163.4 & 19.0 \\
\hline 1.0 & 29.0 & 9.0 & 1.0 & 340.8 & 7.4 & \multirow{2}{*}{\multicolumn{6}{|c|}{ NGC 7145 ; P.A. $132^{\circ}$; Major Axis }} \\
\hline 3.0 & -10.5 & 12.3 & 2.9 & 311.9 & 11.8 & & & & & & \\
\hline 5.5 & -37.5 & 11.5 & 5.3 & 260.1 & 12.0 & 1.3 & -2.9 & 2.2 & 1.3 & 129.7 & 3.6 \\
\hline 13.8 & -31.5 & 18.0 & 14.2 & 244.6 & 25.3 & 4.1 & 14.2 & 3.2 & 4.4 & 117.9 & 6.8 \\
\hline-1.0 & 18.7 & 9.2 & -1.0 & 354.6 & 6.8 & 7.7 & 20.3 & 5.5 & 7.7 & 131.2 & 10.7 \\
\hline-3.0 & 15.1 & 11.0 & -3.0 & 305.9 & 10.7 & 13.3 & 22.3 & 6.8 & 13.9 & 117.4 & 12.9 \\
\hline-5.9 & 33.1 & 13.4 & -5.8 & 269.9 & 14.9 & 21.6 & 41.2 & 14.8 & 23.2 & 121.8 & 22.0 \\
\hline \multirow[t]{3}{*}{-13.3} & 42.5 & 22.5 & -12.5 & 281.6 & 28.0 & -1.1 & -16.0 & 2.3 & -1.1 & 144.1 & 3.5 \\
\hline & & & & & & -4.2 & -19.4 & 3.7 & -4.3 & 135.1 & 6.2 \\
\hline & IC & $96 ;$ P.A & ; Mino & Axis & & -7.7 & -17.0 & 4.4 & -7.6 & 105.3 & 9.3 \\
\hline & & & & & & -12.6 & -23.5 & 5.9 & -14.4 & 101.5 & 13.1 \\
\hline 1.1 & 11.6 & 7.6 & 1.1 & 321.8 & 7.0 & -21.0 & -11.1 & 15.6 & -23.9 & 128.3 & 25.0 \\
\hline 3.1 & 10.6 & 11.0 & 3.1 & 270.9 & 13.4 & & & & & & \\
\hline 5.7 & -3.0 & 17.9 & 5.7 & 274.6 & 20.5 & & NGC & $145 ; \mathrm{P}$ & o; Mino & Axis & \\
\hline 11.2 & -5.5 & 29.8 & $\ldots$ & $\ldots$ & $\ldots$ & & & & & & \\
\hline-0.6 & 14.9 & 7.9 & -0.6 & 345.0 & 7.0 & 1.2 & 0.4 & 2.1 & 1.1 & 125.9 & 3.3 \\
\hline-2.5 & -6.3 & 9.8 & -2.3 & 295.1 & 10.1 & 4.2 & 8.5 & 3.8 & 3.9 & 131.4 & 5.7 \\
\hline-5.1 & -4.2 & 15.1 & -5.1 & 292.7 & 17.0 & 7.5 & 13.9 & 6.8 & 7.2 & 148.6 & 8.9 \\
\hline-11.4 & -4.4 & 31.9 & $\ldots$ & $\ldots$ & $\ldots$ & 13.1 & 19.0 & 7.4 & 15.1 & 129.3 & 10.5 \\
\hline & & & & & & 23.7 & 14.4 & 16.2 & $\ldots$ & $\ldots$ & $\cdots$ \\
\hline & NGC & $5846 ; \mathrm{F}$ & ${ }^{\circ} ; \mathrm{Maj}$ & Axis & & -1.3 & -5.1 & 2.4 & -1.3 & 134.2 & 3.4 \\
\hline 0.5 & -4.0 & 4.0 & 0.5 & 250.8 & 4.2 & $\begin{array}{l}-4.3 \\
-7.7\end{array}$ & $\begin{array}{r}-3.8 \\
-14.4\end{array}$ & $\begin{array}{l}3.9 \\
5.9\end{array}$ & $\begin{array}{l}-4.3 \\
-6.9\end{array}$ & $\begin{array}{l}142.6 \\
124.8\end{array}$ & $\begin{array}{l}7.2 \\
8.3\end{array}$ \\
\hline 2.0 & -21.5 & 8.9 & 2.0 & 220.4 & 10.7 & -12.9 & -7.4 & 7.3 & -13.9 & 124.9 & 10.7 \\
\hline 3.2 & -19.3 & 3.6 & 3.3 & 232.0 & 4.1 & -22.6 & -30.4 & 11.9 & -23.1 & 118.6 & 23.5 \\
\hline 7.3 & -5.1 & 6.2 & 7.4 & 228.6 & 7.3 & & & & & & \\
\hline 15.6 & -0.3 & 6.6 & 15.2 & 208.3 & 8.7 & & IC & 59; P.A & Major & Axis & \\
\hline 0.0 & -19.9 & 7.7 & 0.0 & 249.8 & 8.6 & & & & & & \\
\hline-1.5 & 4.0 & 4.0 & -1.5 & 239.2 & 4.5 & 0.4 & 22.9 & 14.8 & 0.3 & 336.0 & 10.8 \\
\hline-3.3 & 4.9 & 4.9 & -3.3 & 236.7 & 5.6 & 0.7 & -33.3 & 11.4 & 0.6 & 342.3 & 7.9 \\
\hline-6.8 & 3.0 & 4.9 & -6.9 & 225.5 & 5.8 & 1.3 & -46.2 & 8.4 & 1.2 & 333.3 & 8.0 \\
\hline-15.8 & -7.5 & 7.2 & -15.1 & 207.7 & 10.2 & 2.8 & -86.7 & 5.2 & 2.7 & 324.9 & 5.6 \\
\hline & & & & & & 4.9 & -59.5 & 6.3 & 4.8 & 307.8 & 5.3 \\
\hline & NGC & $846 ; \mathrm{P}$ & $0^{\circ} ; \operatorname{Min}$ & I Axis & & 7.7 & -29.8 & 5.8 & 8.0 & 312.8 & 6.4 \\
\hline & & & & & & 12.2 & 7.2 & 6.6 & 12.7 & 271.7 & 7.3 \\
\hline 1.7 & -1.6 & 4.2 & 1.7 & 225.0 & 5.1 & 17.7 & 24.1 & 9.0 & 18.1 & 263.6 & 12.8 \\
\hline 4.5 & -1.6 & 5.1 & 4.5 & 234.9 & 6.0 & 23.9 & 27.8 & 8.7 & 24.0 & 248.7 & 11.4 \\
\hline 9.0 & 5.9 & 7.4 & 8.9 & 228.7 & 8.8 & 38.3 & 85.0 & 11.4 & 33.0 & 245.0 & 23.0 \\
\hline 18.1 & 21.3 & 10.6 & 17.2 & 230.4 & 12.9 & -0.2 & 55.9 & 10.5 & -0.2 & 333.4 & 11.4 \\
\hline-0.3 & 4.3 & 4.3 & -0.3 & 248.6 & 4.7 & -0.7 & 60.6 & 9.8 & -0.8 & 346.7 & 8.7 \\
\hline-2.9 & 7.4 & 3.6 & -2.9 & 227.9 & 4.2 & -1.1 & 99.6 & 12.7 & -1.3 & 340.9 & 7.4 \\
\hline-7.7 & 3.2 & 6.1 & -7.6 & 232.5 & 7.1 & -2.6 & 102.5 & 5.2 & -2.7 & 312.8 & 5.2 \\
\hline-16.7 & 11.4 & 9.0 & -16.1 & 214.0 & 11.7 & -4.9 & 77.4 & 5.4 & -4.7 & 300.9 & 6.8 \\
\hline & & & & & & -8.1 & 27.3 & 5.6 & -7.7 & 298.7 & 6.2 \\
\hline & NGC & $144 ; \mathrm{F}$ & ; Maj & Axis & & -12.8 & -11.6 & 7.3 & -12.1 & 283.9 & 7.7 \\
\hline & & & & & & -17.5 & -41.4 & 9.3 & -17.2 & 256.5 & 11.5 \\
\hline 1.4 & 24.0 & 2.7 & 1.6 & 175.5 & 4.4 & -23.8 & -46.5 & 9.1 & -24.4 & 264.9 & 11.4 \\
\hline 4.2 & 18.9 & 4.4 & 4.4 & 185.0 & 8.3 & -37.2 & -33.2 & 9.6 & -37.2 & 213.7 & 15.3 \\
\hline 7.7 & 30.3 & 5.3 & 7.5 & 143.8 & 10.5 & $\ldots$ & $\ldots$ & $\ldots$ & $\ldots$ & $\ldots$ & $\ldots$ \\
\hline
\end{tabular}


TABLE 7-Continued

\begin{tabular}{|c|c|c|c|c|c|c|c|c|c|c|c|}
\hline$r$ & $v_{r}$ & $\Delta v_{r}$ & $r$ & $\sigma$ & $\Delta \sigma$ & $r$ & $v_{r}$ & $\Delta v_{r}$ & $r$ & $\sigma$ & $\Delta \sigma$ \\
\hline \multicolumn{6}{|c|}{ IC 1459 ; P.A. $129^{\circ}$; Minor Axis } & \multicolumn{6}{|c|}{ NGC 7507 ; P.A. $15^{\circ}$; Minor Axis } \\
\hline 0.6 & 27.3 & 9.3 & 0.6 & 338.0 & 9.3 & 26.7 & 44.0 & 7.3 & 30.8 & 164.9 & 16.8 \\
\hline 1.4 & 7.9 & 10.6 & 1.4 & 317.3 & 10.3 & -1.3 & 6.0 & 2.1 & -1.3 & 225.2 & 3.5 \\
\hline 3.3 & 9.6 & 5.8 & 3.3 & 308.0 & 7.2 & -4.4 & -18.4 & 3.1 & -4.5 & 206.9 & 5.7 \\
\hline 7.0 & -1.5 & 5.4 & 6.4 & 257.5 & 8.5 & -7.7 & -36.6 & 3.9 & -7.9 & 193.0 & 7.6 \\
\hline 13.6 & 8.1 & 8.1 & 12.4 & 219.2 & 14.7 & -14.0 & -36.7 & 4.6 & -13.6 & 189.1 & 9.0 \\
\hline 28.6 & -5.4 & 16.0 & 25.3 & 231.3 & 23.9 & -26.4 & -36.5 & 7.9 & -28.1 & 147.0 & 13.3 \\
\hline-0.5 & 44.2 & 14.0 & -0.6 & 361.8 & 8.8 & & & & & & \\
\hline-1.4 & 29.4 & 9.9 & -1.4 & 331.7 & 9.5 & \multicolumn{6}{|c|}{ NGC 7619; P.A. $34^{\circ}$; Major Axis } \\
\hline-3.5 & -5.1 & 5.7 & -3.4 & 291.0 & 6.8 & & & & & & \\
\hline-7.0 & -9.8 & 5.9 & -6.7 & 268.0 & 9.5 & 0.9 & -38.6 & 3.7 & 0.6 & 323.1 & 5.1 \\
\hline-13.7 & -13.1 & 8.8 & -12.6 & 233.2 & 14.6 & 3.0 & -71.2 & 5.7 & 2.9 & 303.4 & 6.7 \\
\hline-26.5 & -9.3 & 13.2 & -23.9 & 227.2 & 22.6 & 6.2 & -77.8 & 6.5 & 6.1 & 275.0 & 12.7 \\
\hline \multirow{2}{*}{\multicolumn{6}{|c|}{ NGC 7507 ; P.A. $105^{\circ}$; Major Axis }} & 11.6 & -72.6 & 9.3 & 11.7 & 232.1 & 11.9 \\
\hline & & & & & & 24.2 & -38.8 & 12.7 & $\ldots$ & $\ldots$ & \\
\hline 1.4 & -5.7 & 2.8 & 1.4 & 251.3 & 4.3 & -3.0 & $\begin{array}{l}35.4 \\
55.9\end{array}$ & $\begin{array}{l}4.9 \\
5.5\end{array}$ & $\begin{array}{l}-1.1 \\
-2.8\end{array}$ & $\begin{array}{l}341.3 \\
283.9\end{array}$ & $\begin{array}{l}6.1 \\
8.2\end{array}$ \\
\hline 4.3 & -14.4 & 3.6 & 4.3 & 227.9 & 6.5 & -5.7 & 48.6 & 6.0 & -5.4 & 271.2 & 8.9 \\
\hline 7.8 & -17.1 & 4.7 & 7.8 & 225.9 & 8.4 & -10.6 & 65.8 & 7.5 & -9.0 & 202.3 & 13.3 \\
\hline 13.7 & -12.6 & 5.0 & 13.0 & 199.3 & 10.1 & -23.1 & 63.0 & 11.8 & -19.3 & 244.7 & 14.3 \\
\hline 25.9 & -11.7 & 8.3 & 20.9 & 179.4 & 13.9 & & & & & & \\
\hline-1.3 & 20.0 & 2.8 & -1.4 & 236.9 & 4.4 & \multicolumn{6}{|c|}{ NGC 7619 ; P.A. $124^{\circ}$; Minor Axis } \\
\hline-4.4 & 10.1 & 3.7 & -4.4 & 213.3 & 6.8 & & & & & & \\
\hline-7.7 & 0.3 & 4.7 & -8.0 & 187.6 & 7.9 & 0.9 & 25.1 & 3.3 & 0.6 & 318.5 & 3.8 \\
\hline-13.1 & 7.6 & 5.6 & -13.4 & 210.4 & 12.6 & 2.8 & 6.0 & 8.1 & 2.9 & 289.9 & 5.9 \\
\hline-25.7 & 27.7 & 8.8 & -20.6 & 172.0 & 16.8 & 5.4 & 6.1 & 6.4 & 6.5 & 262.8 & 17.4 \\
\hline \multirow{2}{*}{\multicolumn{6}{|c|}{ NGC 7507 ; P.A. $15^{\circ}$; Minor Axis }} & 9.2 & 39.5 & 26.7 & 10.4 & 222.1 & 13.9 \\
\hline & & & & & & 21.4 & -49.4 & 16.1 & 15.2 & 217.1 & 34.1 \\
\hline 1.4 & & & 15 & & & $\begin{array}{r}-1.2 \\
2.8\end{array}$ & 39.0 & 4.1 & $\begin{array}{r}-0.9 \\
20\end{array}$ & 318.5 & $\begin{array}{l}5.0 \\
5.5\end{array}$ \\
\hline 4.2 & $\begin{array}{r}9.1 \\
10.1\end{array}$ & $\begin{array}{l}2.4 \\
3.2\end{array}$ & $\begin{array}{l}1.3 \\
4.4\end{array}$ & $\begin{array}{l}231.4 \\
235.6\end{array}$ & $\begin{array}{l}3.8 \\
5.3\end{array}$ & $\begin{array}{l}-2.0 \\
-5.6\end{array}$ & $\begin{array}{r}18.6 \\
-10.8\end{array}$ & 6.5 & $\begin{array}{l}-3.0 \\
-6.3\end{array}$ & $\begin{array}{l}295.2 \\
292.7\end{array}$ & $\begin{array}{r}5.5 \\
23.9\end{array}$ \\
\hline 7.5 & 33.3 & 3.8 & 8.1 & 210.5 & 7.6 & -21.8 & 19.6 & 14.0 & -10.3 & 234.5 & 10.9 \\
\hline 13.5 & 33.4 & 4.2 & 14.6 & 192.6 & 8.0 & $\ldots$ & $\ldots$ & $\ldots$ & $\ldots$ & $\ldots$ & $\ldots$ \\
\hline
\end{tabular}

\section{REFERENCES}

Bender, R. 1988, Astr. Ap. 202, L5.

Bertola, F., Bettoni, D., Danziger, J., Sadler, E. and de Zeeuw, P. T. 1989, in preparation.

Bertola, F., Bettoni, D., Rusconi, L., and Sedmak, G. 1984, A.J., 89, 356

Bertola, F., Capaccioli, M., Galletta, G., and Rampazzo, R. 1988, Astr. Ap., 192, 24.

Binney, J. J. 1985, M.N.R.A.S., 212, 769.

Birkinshaw, M., and Davies, R. L. 1985, Ap. J., 291, 32.

Burstein, D., Davies, R. L., Dressler, A., Faber, S. M., Stone, R. P. S., Lynden-

Bell, D., Terlevich, R., and Wegner, G. 1987, Ap. J. Suppl., 64, 601.

Canizares, C. R., Fabbiano, G., and Trinchieri, G. 1987, Ap. J., 312, 503.

Davies, R. L., and Birkinshaw, M. 1986, Ap. J. (Letters), 303, L45.

. 1988, Ap. J. Suppl., 68, 409.

Davies, R. L., Burstein, D., Dressler, A., Faber, S. M., Lynden-Bell, D., Terlevitch, R. J. and Wegner, G. 1987, Ap. J. Suppl., 64581.

Davies, R. L., Efstathiou, G., Fall, S. M., Illingworth, G. D., and Schechter, P. L. $1983, A p . J ., 266,41$.

Davies, R. L., and Illingworth, G. D. 1983, Ap. J., 266, 516.

. 1986, Ap.J., 302, 234.

Davis, L. E., Cawson, M., Davies, R. L. and Illingworth, G. 1985, A.J., 90, 169.

de Vaucoulerus, G., de Vaucouleurs, A., and Corwin, H. G. 1976, Second Reference Catalogue of Bright Galaxies (Austin: University of Texas) (RC2).

de Zeeuw, P. T. 1987, in IAU Symposium 127, Structure and Dynamics of

Elliptical Galaxies, ed. T. de Zeeuw (Dordrecht: Reidel), p. 271.

Disney, M. J., and Wall, J. V. 1977, M.N.R.A.S., 179, 235.

Djorgovski, S. 1985, Ph.D. thesis, University of California, Berkeley.

Dressel, L. L., and Condon, J. J. 1978, Ap. J. Suppl., 36, 53.

Franx, M. 1988, Ph.D. thesis, Leiden University.

Franx, M., and de Zeeuw, P. T. 1989, in preparation.

Franx, M., and Illingworth, G. D. 1988, Ap. J. (Letters), 327, L55.

. 1989 , in preparation.
Franx, M., Illingworth, G. D., and Heckman, T. M. 1989, A.J., in press (Paper I).

Franx, M., Illingworth, G. D., and de Zeeuw, P. T. 1989, in preparation (FIZ).

Gerhard, O. E. 1987, in IAU Symposium 127, Structure and Dynamics of Elliptical Galaxies, ed. T. de Zeeuw (Dordrecht: Reidel), p. 241.

Heckman, T. M., Illingworth, G. D., Miley, G. K., and van Breugel, W. J. M. 1985, Ap. J., 299, 41.

Illingworth, G. D., and Franx, M. 1989, in Dynamics of Dense Stellar Systems, ed. D. Merritt (Cambridge: Cambridge University Press), in press.

Jedrzejewski, R. I. 1987, M.N.R.A.S., 226, 747.

Jedrzejewski, R., and Schechter, P. L. 1988, Ap. J. (Letters), 330, L87.

Jura, M., Kim, D. W., Knapp, G. R., and Guhathakurta, P. 1987, Ap. J. (Letters), 312, L11.

Kormendy, J., and Illingworth, G. 1982, Ap. J., 256, 481.

Lauer, T. R. 1985, Ap. J. Suppl., 57, 473.

Norman, C. A., May, A., and van Albada, T. S. 1985, Ap. J., 296, 20.

Peletier, R. F., Illingworth, G. D., Davies, R. L., Davis, L. E., and Cawson, M. C. 1989, A.J., preprint.

Rampazzo, R. 1988, Astr. Ap., 204, 81.

Sandage, A., and Tammann, G. A. 1981, A Revised Shapley Ames Catalogue of Bright Galaxies (Washington: Carnegie Institution of Washington) (RSA).

Sargent, W. L. W., Schechter, P. L., Boksenberg, A., and Shortridge, K. 1977, Ap. J., 212, 326.

Schechter, P. L., and Gunn, J. 1979, Ap. J., 229, 472.

Statler, T. S., 1987, Ap. J., 321, 113.

Tonry, J. L., and Davis, M. 1979, A.J., 84, 1511.

Wagner, S. J., Bender, R., and Möllenhof, C. 1988, Astr. Ap., 195, L5.

Wilkinson, A., and James, R. A. 1982, M.N.R.A.S., 199, 171

Wilkinson, A., Sharples, R. M., Fosbury, R. A. E., and Wallace, P. T. 1986, M.N.R.A.S., $218,297$.

Williams, T. B. 1981, Ap. J., 244, 458.

MariJn FranX: Center for Astrophysics, 60 Garden Street, Cambridge, MA 02138

Timothy Heckman: Space Telescope Science Institute and the Department of Physics and Astronomy, Johns Hopkins University, Baltimore, MD 21218

GARTH IllingwORTH: Lick Observatory, University of California, Santa Cruz, CA 95064 